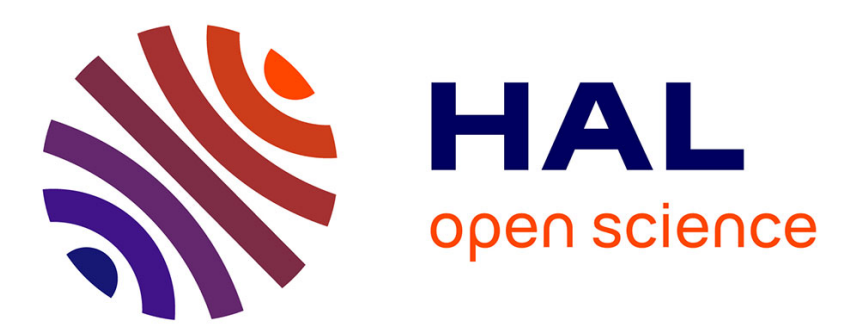

\title{
Speckle observations with PISCO in Merate. VIII. Astrometric measurements of visual binaries in 2007 and new orbits of the multiple system Zeta Aqr
}

\author{
M. Scardia, Jean-Louis Prieur, L. Pansecchi, R.W. Argyle, M. Sala
}

\section{- To cite this version:}

M. Scardia, Jean-Louis Prieur, L. Pansecchi, R.W. Argyle, M. Sala. Speckle observations with PISCO in Merate. VIII. Astrometric measurements of visual binaries in 2007 and new orbits of the multiple system Zeta Aqr. Astronomical Notes / Astronomische Nachrichten, 2010, 000 (331), pp.286 - 299. 10.1002/asna.200911314 . hal-02900250

\section{HAL Id: hal-02900250 \\ https://hal.science/hal-02900250}

Submitted on 15 Jul 2020

HAL is a multi-disciplinary open access archive for the deposit and dissemination of scientific research documents, whether they are published or not. The documents may come from teaching and research institutions in France or abroad, or from public or private research centers.
L'archive ouverte pluridisciplinaire HAL, est destinée au dépôt et à la diffusion de documents scientifiques de niveau recherche, publiés ou non, émanant des établissements d'enseignement et de recherche français ou étrangers, des laboratoires publics ou privés. 


\title{
Speckle observations with PISCO in Merate: VIII. Astrometric measurements of visual binaries in 2007 and new orbits of the multiple system Zeta Aqr
}

\author{
M. Scardia ${ }^{1}$, J.-L. Prieur ${ }^{2}$, L. Pansecchi ${ }^{1}$, R.W. Argyle ${ }^{3}$, and M. Sala ${ }^{1}$ \\ 1 INAF - Osservatorio Astronomico di Brera, Via E. Bianchi 46, 23807 Merate, Italy \\ 2 Laboratoire d'Astrophysique de Toulouse-Tarbes, Université de Toulouse, CNRS, 14 avenue E. Belin, 31400 \\ Toulouse, France \\ 3 Institute of Astronomy, Madingley Road, Cambridge, CB3 0HA, United Kingdom
}

Received May 15th 2010; accepted

Key words Stars: binaries: close - binaries: visual — astrometry — techniques: interferometric — individual (Zeta Aqr, ADS 15971)

\begin{abstract}
We present relative astrometric measurements of visual binaries made during the second semester of 2007, with the speckle camera PISCO at the $102 \mathrm{~cm}$ Zeiss telescope of Brera Astronomical Observatory, in Merate. Our sample contains orbital couples as well as binaries whose motion is still uncertain. We obtained 283 new measurements of 279 objects, with angular separations in the range $0^{\prime \prime} .17-4^{\prime \prime} .4$, and an average accuracy of $0^{\prime \prime} .014$. The mean error on the position angles is $0^{\circ} .6$. Most of the position angles were determined without the usual $180^{\circ}$ ambiguity with the application of triple-correlation techniques and/or by inspection of the long integration files.

We also present the new orbit we have computed for Zeta Aqr AB (ADS 15971), for which our measurements lead to large residuals with the previously computed orbit. We were also able to compute the elements of the perturbation orbit Bb-P caused by an invisible companion, whose mass is estimated at $0.7 \mathrm{M}_{\odot}$.
\end{abstract}

\section{Introduction}

This paper deals with the results of speckle observations of visual binary stars made in Merate (Italy) during the second semester of 2007 with the Pupil Interferometry Speckle camera and COronagraph (PISCO) on the $102 \mathrm{~cm}$ Zeiss telescope of INAF - Osservatorio Astronomico di Brera (OAB, Brera Astronomical Observatory). It is the eighth of a series (Scardia et al. 2005, 2006, 2007, 2008b, Prieur et al. 2008, Scardia et al. 2009, Prieur et al., 2009, herein: Papers I to VII), whose purpose is to contribute to the determination of binary orbits. PISCO was developed at Observatoire Midi-Pyrénées (France) and first used at Pic du Midi from 1993 to 1998. It was moved to Merate in 2003 and used there since.

We briefly describe our observations in Sect. 2 . Then we present and discuss the astrometric measurements in Sect. 3. Finally in Sect. 4 we propose new revised orbits for the multiple system Zeta Aqr and derive estimate values for the component masses.

\section{Observations and description of the sample}

The observations were carried out with the PISCO speckle camera with the ICCD (Intensified Charge
Coupled Device) detector belonging to Nice University (France). This instrumentation is presented in Prieur et al. (1998) and our observing procedure is described in detail in Paper VI. For the present observations, thanks to an improvement of our software and a faster computer, we were also able to compute in real time the restricted triple correlation (Aristidi et al., 1997) that we use for resolving the $180^{\circ}$ ambiguity (see Sect. 3.2).

The description of our sample can be found in our previous papers (e.g., Paper VI). It basically includes all the visual binaries for which new measurements are needed to improve their orbits, that are accessible with our instrumentation.

The distribution of the angular separations measured in this paper is displayed in Fig. 1a and shows a maximum for $\rho \approx 0^{\prime \prime} .7$. The largest separation of $\rho=4^{\prime \prime} .4$ was obtained for ADS 11178. The smallest separation was measured for KUI 93, with $\rho=0^{\prime \prime} .17$. This is close to the diffraction limit $\rho_{d}=\lambda / D \approx 0^{\prime \prime} .13$ with the $R$ filter (i.e. $\lambda=650 \mathrm{~nm}$ ) and the Zeiss telescope whose aperture is $D=1.02 \mathrm{~m}$.

The distribution of the apparent magnitudes $m_{V}$ of the binaries measured in this paper is presented in Fig. $1 \mathrm{~b}$ and the difference of magnitudes $\Delta m_{V}$ between the two components in Fig. 1c. The telescope aperture and detector sensitivity lead to a limiting magnitude of about $m_{V}=9$. A careful handling of the image 
processing enabled us to measure a few couples with $\Delta m_{V}>3$ (Fig. 1c).

Using the Hipparcos parallaxes, we were able to construct the HR diagram of those binaries that is displayed in Fig. 2. We only plotted the objects for which the relative uncertainty on the parallax was smaller than $50 \%$. It can be seen that a large part of the HR diagram is covered by our sample. In the future we would like to acquire a more sensitive detector in order to observe fainter (and cooler) main sequence stars.

\section{Astrometric measurements}

The astrometric measurements of the observations made during the second semester of 2007 are displayed in Table 1. The format of the first ten columns of this table is the same as for the previous papers of this series. It is described for instance in Paper VI. In Col. 11, we report some information about the secondary peaks of the auto-correlation files: diffuse (Diff.) or elongated (Elon.). The last three columns are dealing with the residuals and will be presented in Sect. 3.3.

The characteristics of the PISCO $R$ and $V$ filters can be found in Table 1 of Paper III. Some objects were observed with no filter because they were too faint. This is indicated with $W$ (for "white" light) in the filter column (Col. 5). The corresponding bandpass is $420 \mathrm{~nm}$ corresponding to that of the ICCD detector, with a central wavelength of about $650 \mathrm{~nm}$, close to that of the $R$ filter.

The average values of the errors of the 283 measurements reported in this table are $0^{\prime \prime} .014 \pm 0^{\prime \prime} .009$ and $0^{\circ} .56 \pm 0^{\circ} .35$ for $\rho$ and $\theta$, respectively. Our error determination procedure is described in detail in $\mathrm{Pa}$ per III.

We have noticed some peculiarities in the autocorrelations of ADS 504, 11579, and 16011, with the presence of small symmetric patches (that may be due to detector noise ?). We will re-observe those objects in order to verify that those peculiarities are artefacts.

\subsection{The triple system ADS 11344}

ADS 11344 is a triple star whose components A, B and $\mathrm{C}$ give rise, in the literature, to three different systems: HU 66AB, HU 66BC and STT 351AC (also reported as STT 351AB-C). In fact the C component is the brightest star and $B$ the faintest. As the separation of the $\mathrm{AB}$ system is very small, many observers are unable to resolve it and confine themselves to measure $A B$, as it was a single star $(A)$, with respect to C. This is a source of confusion in the literature ${ }^{1}$.In

1 The last reported observation of STT $351 \mathrm{AC}$ in IC4 made in 2005.594 by WSI2006b, with $\rho=0^{\prime \prime} .58$ and $\theta=28 .^{\circ} 5$ seems suspicious. It is inconsistent with the trend of the previous observations, especially for $\rho$. many cases the reported measurements of STT 351AC are in fact those of STT 351AB-C, when the AB system was not resolved. This also happened to us for our first observation of ADS 11344 made in 2004.690 with ( $\left.\rho=0^{\prime \prime} .802, \theta=25 .^{\circ} 7\right)$, reported in Paper II, and wrongly attributed to the STT $351 \mathrm{AC}$ system.

The duplicity of the auto-correlation secondary peaks is clear in the observation of ADS 11344 made in 2007.671 (see Fig. 3). We measured them separately and found that the innermost peaks correspond to STT 351AC and the outermost ones to HU 66BC. Finally, each couple composed by the big and small secondary peaks correspond to HU 66AB. This identification is validated with the small residuals that we obtain with Novakovic (2008b)'s orbit of HU 66BC, and with Seymour (2002)'s orbit of HU 66AB (see Table 1).

\subsection{Quadrant determination}

As our measurements were obtained from the symmetric auto-correlation files, the $\theta$ values first presented a $180^{\circ}$ ambiguity. To try resolving this ambiguity and determine the quadrant containing the companion, we have used Aristidi et al. (1997)'s method by computing and analysing restricted triple correlation files. For the couples with the largest separations, a straightforward determination could be done when the companions were visible in the long integration files.

As a result, in Table 1, we are able to give the non-ambiguous (or "absolute") position angles of 213 out of 283 measurements, i.e. $76 \%$ of the total. They are marked with an asterisk in Col 9. Otherwise, our angular measurements were reduced to the quadrant reported in the "Washington Double Star Catalogue" (Mason et al, 2009, hereafter WDS Catalogue).

Our "absolute" $\theta$ values are consistent with the values tabulated in the WDS Catalogue for all objects except for ADS 10, 10905, 11558, 15954, 16204, 16307, 16958 and 17122 . In all those cases, however, a clear and high contrast between the two secondary peaks of the triple correlation is observed, which is a good indication of the validity of our quadrant determination. In the following, we discuss each case using the usual convention of numbering the quadrants (Q) from 1 to 4 to indicate the North-East, South-East, South-West and North-West quadrants, respectively.

ADS 10: Our value of $Q=2$ is consistent both with the space-based Tycho measurement of 1991.75 (Høg et al. 2000) and with Douglass et al. (1997)'s measurement of 1992.878 . It is likely that the quadrant $\mathrm{Q}=4$ reported in the WDS Catalogue comes from the other 12 observations listed in the "Fourth Catalogue of Interferometric Measurements of Binary Stars" (Hartkopf et al. 2009, hereafter IC4). The two components of ADS 10 have a similar brightness, with $\Delta m_{V}=0.16$, which well accounts for the difficulty of determining the quadrant. Furthermore, most of those observations of IC4 
Table 1 Measurements of binaries with PISCO and residuals with published orbits (begin).

\begin{tabular}{|c|c|c|c|c|c|c|c|c|c|c|c|c|}
\hline WDS & Name & ADS & Epoch & Fil. & $\begin{array}{l}\text { Eyep. } \\
(\mathrm{mm})\end{array}$ & $\begin{array}{c}\rho \\
(")\end{array}$ & $\begin{array}{l}\sigma_{\rho} \\
(")\end{array}$ & $\begin{array}{ll}\theta & \sigma_{\theta} \\
\left(^{\circ}\right) & \left(^{\circ}\right)\end{array}$ & Notes & Orbit & $\begin{array}{r}\Delta \rho(\mathrm{O}-\mathrm{C}) \\
(")\end{array}$ & $\begin{array}{r}\Delta \theta(\mathrm{O}-\mathrm{C}) \\
\left({ }^{\circ}\right)\end{array}$ \\
\hline $00029+4715$ & A 800 & 10 & 2007.967 & W & 20 & 1.584 & 0.010 & $113.3^{*} 0.9$ & & & & \\
\hline $00093+7943$ & STF 2 & 102 & 2007.969 & $\mathrm{R}$ & 20 & 0.825 & 0.008 & $18.0^{*} 0.3$ & & Hei1997 & -0.02 & 1.1 \\
\hline $00116+5558$ & STF 7 & 143 & 2007.967 & $\mathrm{R}$ & 20 & 1.311 & 0.024 & $211.4 \quad 0.6$ & & & & \\
\hline $00214+6700$ & STT $6 \mathrm{AB}$ & 293 & 2007.969 & $\mathrm{R}$ & 20 & 0.653 & 0.008 & $154.4^{*} 0.6$ & & Sca2000b & -0.02 & -0.1 \\
\hline$=4$ & & $"$ & (1) & $"$ & $"$ & $"$ & 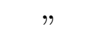 & $" \quad "$ & & Ole2001 & 0.04 & 1.1 \\
\hline $00287+3718$ & A $1504 \mathrm{AB}$ & 382 & 2007.972 & $\mathrm{R}$ & 20 & 0.593 & 0.008 & $42.9 \quad 1.0$ & & & & \\
\hline $00366+5609$ & A 914 & 504 & 2007.969 & $\mathrm{R}$ & 20 & 0.440 & 0.008 & $22.6 \quad 1.0$ & & Nov2008a & -0.02 & -2.8 \\
\hline $00373+5801$ & BU 1097 & 515 & 2007.969 & $\mathrm{R}$ & 20 & 0.510 & 0.009 & $72.5^{*} 0.5$ & & & & \\
\hline $00444+3337$ & STF 55 & 618 & 2007.969 & $\mathrm{~W}$ & 20 & 2.212 & 0.011 & $329.7^{*} 0.3$ & & & & \\
\hline $00480+5127$ & STF 59AB & 659 & 2007.969 & $\mathrm{~W}$ & 20 & 2.251 & 0.013 & $148.0^{*} 0.3$ & & & & \\
\hline $00550+2338$ & STF 73AB & 755 & 2007.972 & $\mathrm{R}$ & 20 & 1.011 & 0.008 & $319.8^{*} 0.6$ & & Doc1990b & -0.02 & -0.9 \\
\hline $01041+2635$ & COU 351 & - & 2007.972 & $\mathrm{~W}$ & 20 & 0.797 & 0.014 & $243.7 \quad 0.4$ & & & & \\
\hline $01089+4512$ & $\mathrm{AC} 13 \mathrm{AB}$ & 936 & 2007.964 & $\mathrm{~W}$ & 20 & 0.595 & 0.009 & $263.3^{*} 0.9$ & & & & \\
\hline $01213+1132$ & $\mathrm{BU} 4 \mathrm{AB}$ & 1097 & 2007.964 & $\mathrm{R}$ & 20 & 0.594 & 0.016 & $107.7^{*} 1.0$ & & Sca2001d & 0.06 & -1.0 \\
\hline $01360+0739$ & STF $138 \mathrm{AB}$ & 1254 & 2007.972 & $\mathrm{R}$ & 20 & 1.717 & 0.012 & $58.2^{*} 0.3$ & & & & \\
\hline $01501+2217$ & STF 174 & 1457 & 2007.964 & $\mathrm{R}$ & 20 & 2.841 & 0.041 & $164.6^{*} 0.3$ & & & & \\
\hline $02211+2956$ & А 962 & 1792 & 2007.964 & $\mathrm{~W}$ & 20 & 0.863 & 0.008 & $66.6^{*} 0.3$ & & & & \\
\hline $14455+4223$ & STT $285 \mathrm{AB}$ & 9378 & 2007.542 & $\mathrm{R}$ & 20 & 0.472 & 0.016 & $92.5 \quad 0.5$ & & Cou1973b & -0.01 & -0.2 \\
\hline $14484+2422$ & STF1884 & 9389 & 2007.542 & $\mathrm{R}$ & 20 & 2.161 & 0.011 & $54.9^{*} 0.3$ & & & & \\
\hline $14497+0759$ & A $1110 \mathrm{AB}$ & 9400 & 2007.543 & $\mathrm{R}$ & 20 & 0.683 & 0.008 & $243.5^{*} 0.8$ & & & & \\
\hline $14497+4843$ & STF1890 & 9406 & 2007.537 & $\mathrm{R}$ & 20 & 2.692 & 0.013 & $45.1^{*} 0.3$ & & & & \\
\hline $15018-0008$ & BU 348AB & 9480 & 2007.545 & $\mathrm{R}$ & 10 & 0.487 & 0.003 & $108.0^{*} 0.3$ & & & & \\
\hline $15038+4739$ & STF1909 & 9494 & 2007.534 & $\mathrm{R}$ & 20 & 1.842 & 0.009 & $57.9^{*} 0.3$ & & Sod1999 & 0.01 & 0.1 \\
\hline $15056+1138$ & STF1907 & 9498 & 2007.537 & $\mathrm{~W}$ & 20 & 0.913 & 0.008 & $\begin{array}{ll}350.6 & 0.3\end{array}$ & & & & \\
\hline $15075+0914$ & STF1910 & 9507 & 2007.567 & $\mathrm{R}$ & 20 & 4.038 & 0.031 & $211.2^{*} 0.3$ & & & & \\
\hline $15116+1007$ & A 1116 & 9530 & 2007.545 & $\mathrm{~W}$ & 20 & 0.808 & 0.008 & $50.9^{*} 0.5$ & & & & \\
\hline $15183+2650$ & STF1932AB & 9578 & 2007.534 & $\mathrm{R}$ & 20 & 1.621 & 0.010 & $262.1^{*} 0.5$ & & Hei1965c & -0.01 & -0.4 \\
\hline $15210+2104$ & HU 146 & 9600 & 2007.534 & $\mathrm{R}$ & 20 & 0.677 & 0.039 & $125.0^{*} 1.7$ & & & & \\
\hline $15232+3017$ & STF1937AB & 9617 & 2007.534 & $\mathrm{R}$ & 10 & 0.510 & 0.003 & $139.6^{*} 0.3$ & & WSI2006b & -0.01 & -1.3 \\
\hline $15245+3723$ & STF1938Ba,Bb & 9626 & 2007.515 & $\mathrm{R}$ & 20 & 2.278 & 0.011 & $6.5^{*} 0.4$ & & Sod1999 & 0.03 & 0.4 \\
\hline $15246+5413$ & HU 149 & 9628 & 2007.543 & $\mathrm{R}$ & 20 & 0.663 & 0.008 & $270.5^{*} 0.3$ & & & & \\
\hline $15261+1810$ & STF1940 & 9634 & 2007.567 & $\mathrm{R}$ & 20 & 0.415 & 0.008 & $331.3 \quad 1.0$ & & & & \\
\hline $15264+4400$ & STT 296AB & 9639 & 2007.545 & $\mathrm{R}$ & 20 & 2.091 & 0.011 & $275.4^{*} 0.5$ & & & & \\
\hline $15277+0606$ & STF1944 & 9647 & 2007.545 & $\mathrm{R}$ & 20 & 0.679 & 0.013 & 299.10 .6 & & & & \\
\hline $15278+2906$ & JEF 1 & - & 2007.515 & $\mathrm{R}$ & 10 & 0.288 & 0.003 & $144.2^{*} 0.3$ & & Tok1984 & 0.01 & 3.1 \\
\hline $15300+2530$ & STF1950 & 9675 & 2007.570 & $\mathrm{R}$ & 20 & 3.297 & 0.016 & $90.7^{*} 0.5$ & & & & \\
\hline $15329+3122$ & COU 610 & - & 2007.515 & $\mathrm{R}$ & 10 & 0.815 & 0.004 & $199.1^{*} 0.3$ & & & & \\
\hline $15348+1032$ & STF1954AB & 9701 & 2007.551 & $\mathrm{R}$ & 20 & 4.025 & 0.020 & $172.8^{*} 0.3$ & & WSI2004a & 0.03 & -0.1 \\
\hline $15360+3948$ & STT 298AB & 9716 & 2007.551 & $\mathrm{R}$ & 20 & 0.979 & 0.008 & $175.4^{*} 0.3$ & & Sod1999 & -0.02 & 0.5 \\
\hline $15361+4849$ & HU 652 & 9718 & 2007.515 & $\mathrm{R}$ & 20 & 1.106 & 0.014 & $184.3 \quad 0.6$ & & & & \\
\hline $15396+7959$ & STF1989 & 9769 & 2007.551 & $\mathrm{R}$ & 20 & 0.670 & 0.008 & $25.3^{*} 0.3$ & & Hrt2008 & 0.04 & 1.3 \\
\hline $15405+1840$ & A 2076 & 9742 & 2007.537 & $\mathrm{R}$ & 20 & 0.716 & 0.008 & $\begin{array}{ll}184.1 & 0.7\end{array}$ & & & & \\
\hline $15413+5959$ & STF1969 & 9756 & 2007.543 & $\mathrm{~W}$ & 20 & 0.955 & 0.008 & $27.4^{*} 0.4$ & & Hei1975b & 0.09 & -0.7 \\
\hline $15427+2618$ & STF1967 & 9757 & 2007.537 & $\mathrm{R}$ & 10 & 0.697 & 0.006 & $112.9^{*} 0.3$ & & Hrt1989 & -0.03 & 0.2 \\
\hline $15432+1340$ & BU 619 & 9758 & 2007.570 & $\mathrm{R}$ & 20 & 0.671 & 0.009 & $1.3^{*} 0.9$ & Diff. & & & \\
\hline $15487+8337$ & STF2034 & 9853 & 2007.551 & $\mathrm{R}$ & 20 & 1.106 & 0.009 & $109.4^{*} 0.4$ & & & & \\
\hline $15498+4431$ & BU 621 & 9802 & 2007.583 & $\mathrm{R}$ & 20 & 0.672 & 0.010 & $28.1^{*} 1.0$ & & & & \\
\hline $15509+1911$ & A 2078 & 9809 & 2007.581 & $\mathrm{~W}$ & 20 & 1.060 & 0.008 & $162.6^{*} 0.4$ & & & & \\
\hline $15568+1229$ & STF1988 & 9850 & 2007.581 & $\mathrm{R}$ & 20 & 1.885 & 0.010 & $250.6^{*} 0.6$ & & & & \\
\hline $16009+1316$ & STT $303 \mathrm{AB}$ & 9880 & 2007.515 & $\mathrm{R}$ & 20 & 1.533 & 0.011 & $172.1^{*} 0.3$ & & & & \\
\hline $16030+1359$ & STF2000 & 9904 & 2007.534 & $\mathrm{R}$ & 20 & 2.564 & 0.013 & $227.1^{*} 0.3$ & & & & \\
\hline $16133+1332$ & STF2021AB & 9969 & 2007.551 & $\mathrm{R}$ & 20 & 4.107 & 0.021 & $355.6^{*} 0.3$ & & Hop1964b & 0.02 & 0.9 \\
\hline $16135+7147$ & A 1136 & 9985 & 2007.570 & $\mathrm{~W}$ & 20 & 0.727 & 0.011 & $8.6^{*} 1.0$ & & & & \\
\hline $16137+4638$ & A 1642 & 9975 & 2007.534 & $\mathrm{R}$ & 20 & 0.753 & 0.009 & $182.1^{*} 0.5$ & & Hrt2001b & 0.03 & -0.5 \\
\hline $16145+0531$ & STF2023 & 9974 & 2007.534 & $\mathrm{R}$ & 20 & 1.925 & 0.010 & $223.8^{*} 0.3$ & & & & \\
\hline
\end{tabular}


Table 1 - continued

\begin{tabular}{|c|c|c|c|c|c|c|c|c|c|c|c|c|c|}
\hline WDS & Name & $\mathrm{ADS}$ & Epoch & Fil. & $\begin{array}{l}\text { Eyep. } \\
(\mathrm{mm})\end{array}$ & $\begin{array}{c}\rho \\
(")\end{array}$ & $\begin{array}{l}\sigma_{\rho} \\
(")\end{array}$ & $\begin{array}{l}\theta \\
\left({ }^{\circ}\right)\end{array}$ & $\begin{array}{l}\sigma_{\theta} \\
\left(^{\circ}\right)\end{array}$ & Notes & Orbit & $\begin{array}{r}\Delta \rho(\mathrm{O}-\mathrm{C}) \\
(")\end{array}$ & $\begin{array}{r}\Delta \theta(\mathrm{O}-\mathrm{C}) \\
\left({ }^{\circ}\right)\end{array}$ \\
\hline $16153+0416$ & STF2027 & 9980 & 2007.534 & $\mathrm{R}$ & 20 & 1.894 & 0.019 & 80.8 & 0.4 & & & & \\
\hline $16160+0721$ & STF2026 & 9982 & 2007.537 & $\mathrm{~W}$ & 20 & 3.385 & 0.017 & $17.9^{*}$ & 0.3 & & Hei1963a & 0.00 & -0.5 \\
\hline $16231+4738$ & STF2047 & 10038 & 2007.545 & $\mathrm{R}$ & 20 & 1.836 & 0.019 & 324.1 & 0.3 & & & & \\
\hline $16238+6142$ & STF2054AB & 10052 & 2007.543 & $\mathrm{R}$ & 20 & 0.985 & 0.008 & $351.1^{*}$ & 0.3 & & & & \\
\hline $16279+2559$ & STF2049 & 10070 & 2007.551 & $\mathrm{R}$ & 20 & 1.125 & 0.008 & $194.6^{*}$ & 0.5 & & & & \\
\hline $16289+1825$ & STF2052AB & 10075 & 2007.543 & $\mathrm{R}$ & 20 & 2.209 & 0.011 & $121.3^{*}$ & 0.3 & & Sod1999 & 0.05 & -0.0 \\
\hline $16309+0159$ & STF2055AB & 10087 & 2007.543 & $\mathrm{~V}$ & 20 & 1.413 & 0.008 & $35.3^{*}$ & 0.3 & & Hei1993b & -0.04 & 0.2 \\
\hline $16309+3804$ & STF2059 & 10093 & 2007.551 & $\mathrm{R}$ & 20 & 0.376 & 0.012 & 185.9 & 1.0 & & & & \\
\hline $16326+4007$ & STT 313 & 10111 & 2007.551 & $\mathrm{R}$ & 20 & 0.931 & 0.008 & $130.2^{*}$ & 0.3 & & & & \\
\hline $16362+5255$ & STF2078AB & 10129 & 2007.567 & $\mathrm{R}$ & 20 & 3.151 & 0.016 & $104.0^{*}$ & 0.3 & & & & \\
\hline $16438+5133$ & HU 664 & 10189 & 2007.567 & $\mathrm{R}$ & 20 & 0.498 & 0.009 & 303.2 & 1.4 & & & & \\
\hline $16448+3544$ & STF2097AB & 10193 & 2007.570 & $\mathrm{~W}$ & 20 & 1.930 & 0.017 & $79.2^{*}$ & 0.6 & & & & \\
\hline $16458+3538$ & STF2101AB & 10203 & 2007.594 & $\mathrm{R}$ & 20 & 4.104 & 0.021 & $47.7^{*}$ & 0.3 & & & & \\
\hline $16483+0244$ & BU 43 & 10217 & 2007.581 & $\mathrm{~W}$ & 20 & 1.373 & 0.014 & $55.5^{*}$ & 0.4 & & & & \\
\hline $16514+0113$ & STT 315 & 10230 & 2007.567 & $\mathrm{R}$ & 20 & 0.633 & 0.008 & $314.0^{*}$ & 0.3 & & Doc2007d & -0.00 & 0.1 \\
\hline $16540+2906$ & A 350 & 10253 & 2007.581 & $\mathrm{~W}$ & 20 & 0.580 & 0.014 & $146.8^{*}$ & 1.0 & & & & \\
\hline $16564+6502$ & STF2118AB & 10279 & 2007.545 & $\mathrm{R}$ & 20 & 1.054 & 0.008 & 66.5 & 0.3 & & Sca2002d & -0.10 & -0.9 \\
\hline $16567+1408$ & STT 318 & 10270 & 2007.551 & $\mathrm{~W}$ & 20 & 2.811 & 0.014 & $242.9^{*}$ & 0.3 & & & & \\
\hline $16581+1509$ & STT 319 & 10277 & 2007.567 & $\mathrm{R}$ & 20 & 0.847 & 0.008 & 64.3 & 0.8 & & & & \\
\hline $16588+0358$ & STF3107AB & 10285 & 2007.545 & $\mathrm{~W}$ & 20 & 1.443 & 0.008 & $73.1^{*}$ & 0.4 & & & & \\
\hline $16594+1419$ & STT 321 & 10294 & 2007.567 & $\mathrm{~W}$ & 20 & 0.584 & 0.008 & $14.2^{*}$ & 0.9 & & & & \\
\hline $17020+0827$ & STF2114 & 10312 & 2007.690 & $\mathrm{R}$ & 20 & 1.334 & 0.014 & $194.4^{*}$ & 0.3 & & & & \\
\hline $17047+1936$ & PRY 2AB & 10326 & 2007.592 & $\mathrm{R}$ & 20 & 1.843 & 0.009 & $228.2^{*}$ & 0.3 & & & & \\
\hline $17053+5428$ & STF2130AB & 10345 & 2007.567 & $\mathrm{R}$ & 20 & 2.363 & 0.012 & $10.6^{*}$ & 0.3 & & Hei1981b & 0.04 & 1.8 \\
\hline $17082-0105$ & A 1145 & 10355 & 2007.592 & $\mathrm{R}$ & 20 & 0.658 & 0.011 & $349.0^{*}$ & 0.7 & & WSI2006b & 0.02 & 0.3 \\
\hline $17096+0356$ & HEI 894 & - & 2007.592 & $\mathrm{R}$ & 20 & 0.549 & 0.012 & 21.7 & 1.3 & & & & \\
\hline $17131+5408$ & STF2146AB & 10410 & 2007.581 & $\mathrm{~W}$ & 20 & 2.680 & 0.017 & $224.4^{*}$ & 0.4 & & & & \\
\hline $17166-0027$ & A 2984 & 10429 & 2007.592 & $\mathrm{R}$ & 20 & 0.839 & 0.008 & $11.7^{*}$ & 0.5 & & Ole1993 & -0.19 & -0.3 \\
\hline $17237+3709$ & STF2161Aa-B & 10526 & 2007.594 & $\mathrm{R}$ & 20 & 4.036 & 0.020 & $319.5^{*}$ & 0.3 & & & & \\
\hline $17237+3709$ & $\mathrm{MCA} 48 \mathrm{Aa}, \mathrm{Ab}$ & 10526 & 2007.594 & $\mathrm{R}$ & 10 & 0.218 & 0.013 & 31.7 & 3.6 & Diff. & & & \\
\hline $17240-0050$ & STF2156 & 10518 & 2007.594 & $\mathrm{~W}$ & 20 & 3.769 & 0.019 & $35.9^{*}$ & 0.3 & Elon. & & & \\
\hline $17246+1536$ & STF2160 & 10528 & 2007.668 & $\mathrm{R}$ & 20 & 3.780 & 0.019 & $65.9^{*}$ & 0.3 & Diff. & & & \\
\hline $17266+3546$ & STF2168 & 10558 & 2007.581 & W & 20 & 2.319 & 0.012 & $201.2^{*}$ & 0.3 & & & & \\
\hline $17290+5052$ & STF2180 & 10597 & 2007.567 & $\mathrm{R}$ & 20 & 3.041 & 0.018 & $259.3^{*}$ & 0.3 & & & & \\
\hline $17320+0249$ & $\mathrm{STT} 331 \mathrm{AB}$ & 10614 & 2007.584 & $\mathrm{~W}$ & 20 & 1.013 & 0.008 & $349.8^{*}$ & 0.3 & & & & \\
\hline $17350+6153$ & BU 962AB & 10660 & 2007.655 & $\mathrm{R}$ & 20 & 1.222 & 0.008 & $322.0^{*}$ & 0.3 & & Sod1999 & -0.05 & 0.0 \\
\hline $17400-0038$ & BU 631 & 10696 & 2007.671 & $\mathrm{R}$ & 10 & 0.260 & 0.005 & 89.8 & 0.5 & & Hei1996a & -0.01 & 3.9 \\
\hline $17403+6341$ & STF2218 & 10728 & 2007.595 & $\mathrm{R}$ & 20 & 1.494 & 0.008 & $312.4^{*}$ & 0.3 & & & & \\
\hline $17412+4139$ & STF2203 & 10722 & 2007.567 & $\mathrm{R}$ & 20 & 0.743 & 0.009 & 294.0 & 0.8 & & & & \\
\hline $17434+3357$ & HO 560 & 10742 & 2007.592 & W & 20 & 1.320 & 0.008 & $262.9^{*}$ & 0.3 & & & & \\
\hline $17464+0542$ & STF2212 & 10779 & 2007.671 & $\mathrm{~W}$ & 20 & 3.226 & 0.016 & $340.4^{*}$ & 0.3 & & & & \\
\hline $17506+0714$ & STT 337 & 10828 & 2007.592 & $\mathrm{R}$ & 20 & 0.541 & 0.009 & 168.2 & 1.3 & & Doc1990a & 0.04 & 2.0 \\
\hline $17512+4454$ & STF2242 & 10849 & 2007.655 & $\mathrm{R}$ & 20 & 3.387 & 0.017 & 326.0 & 0.3 & & & & \\
\hline $17520+1520$ & STT $338 \mathrm{AB}$ & 10850 & 2007.655 & $\mathrm{R}$ & 20 & 0.825 & 0.008 & 165.5 & 0.3 & & & & \\
\hline $17533+4000$ & BU 130 & 10875 & 2007.655 & $\mathrm{R}$ & 20 & 1.579 & 0.016 & $110.6^{*}$ & 0.3 & & & & \\
\hline $17541+2949$ & $\mathrm{AC} 9$ & 10880 & 2007.655 & $\mathrm{~W}$ & 20 & 1.097 & 0.008 & $241.1^{*}$ & 0.4 & Elon. & & & \\
\hline $17564+1820$ & STF2245AB & 10905 & 2007.655 & $\mathrm{R}$ & 20 & 2.623 & 0.013 & $111.1^{*}$ & 0.3 & & & & \\
\hline $18014+6557$ & STF2284 & 11016 & 2007.685 & $\mathrm{R}$ & 20 & 3.594 & 0.023 & $191.5^{*}$ & 0.3 & Diff. & & & \\
\hline $18018+0118$ & BU $1125 \mathrm{AB}$ & 10990 & 2007.668 & $\mathrm{R}$ & 10 & 0.508 & 0.003 & $135.4^{*}$ & 0.6 & & Pop2000a & -0.08 & -2.3 \\
\hline $18070+3034$ & $\mathrm{AC} 15 \mathrm{AB}$ & 11077 & 2007.668 & $\mathrm{R}$ & 20 & 1.008 & 0.013 & $305.2^{*}$ & 0.3 & & Sod1999 & 0.03 & 1.8 \\
\hline $18096+0609$ & STF2283 & 11110 & 2007.668 & $\mathrm{R}$ & 20 & 0.609 & 0.008 & 56.2 & 1.6 & & & & \\
\hline $18113+6915$ & STF2307 & 11178 & 2007.685 & W & 20 & 4.398 & 0.071 & $205.4^{*}$ & 0.3 & & & & \\
\hline $18118+3327$ & $\mathrm{HO} 82 \mathrm{AB}-\mathrm{C}$ & 11149 & 2007.668 & $\mathrm{R}$ & 20 & 0.712 & 0.011 & $218.4^{*}$ & 0.7 & & & & \\
\hline $18121+2739$ & STF2292 & 11155 & 2007.671 & $\mathrm{R}$ & 20 & 0.899 & 0.008 & 273.9 & 0.5 & & & & \\
\hline
\end{tabular}


Table 1 - continued

\begin{tabular}{|c|c|c|c|c|c|c|c|c|c|c|c|c|c|}
\hline WDS & Name & $\mathrm{ADS}$ & Epoch & Fil. & $\begin{array}{l}\text { Eyep. } \\
(\mathrm{mm})\end{array}$ & $\begin{array}{c}\rho \\
(")\end{array}$ & $\begin{array}{l}\sigma_{\rho} \\
(")\end{array}$ & $\begin{array}{l}\theta \\
\left({ }^{\circ}\right)\end{array}$ & $\begin{array}{l}\sigma_{\theta} \\
\left(^{\circ}\right)\end{array}$ & Notes & Orbit & $\begin{array}{r}\Delta \rho(\mathrm{O}-\mathrm{C}) \\
(")\end{array}$ & $\begin{array}{r}\Delta \theta(\mathrm{O}-\mathrm{C}) \\
\left({ }^{\circ}\right)\end{array}$ \\
\hline $18126-0329$ & A 83 & 11154 & 2007.690 & $\mathrm{~W}$ & 20 & 0.752 & 0.014 & 303.4 & 0.8 & & & & \\
\hline $18126+3836$ & BU 1091 & 11170 & 2007.668 & $\mathrm{~W}$ & 20 & 0.719 & 0.008 & $320.5^{*}$ & 0.6 & & & & \\
\hline $18218+2130$ & BU 641 & 11287 & 2007.671 & $\mathrm{R}$ & 20 & 0.798 & 0.008 & $341.0^{*}$ & 0.6 & & & & \\
\hline $18239+5848$ & STF2323AB & 11336 & 2007.685 & $\mathrm{R}$ & 20 & 3.757 & 0.056 & $348.5^{*}$ & 0.3 & Diff. & Nov2006e & 0.01 & -0.3 \\
\hline $18253+4846$ & $\mathrm{STT} 351 \mathrm{AC}$ & 11344 & 2007.671 & $\mathrm{R}$ & 20 & 0.753 & 0.011 & 24.6 & 0.8 & & & & \\
\hline $18253+4846$ & HU 66BC & 11344 & 2007.671 & $\mathrm{R}$ & 20 & 0.928 & 0.008 & 28.0 & 0.6 & & Nov2008b & 0.02 & 0.1 \\
\hline $18253+4846$ & HU $66 \mathrm{AB}$ & 11344 & 2007.671 & $\mathrm{R}$ & 20 & 0.183 & 0.011 & 222.5 & 2.2 & & Sey2002 & -0.02 & -0.4 \\
\hline $18269+0625$ & STT $350 \mathrm{AB}$ & 11349 & 2007.687 & $\mathrm{R}$ & 20 & 1.853 & 0.011 & $165.7^{*}$ & 0.4 & & & & \\
\hline $18278+2442$ & STF2320 & 11373 & 2007.688 & $\mathrm{R}$ & 20 & 1.119 & 0.011 & $359.4^{*}$ & 0.3 & & & & \\
\hline $18295+2955$ & STF2328AB & 11397 & 2007.688 & $\mathrm{~W}$ & 20 & 3.698 & 0.018 & $71.4^{*}$ & 0.3 & & & & \\
\hline $18310+0123$ & STF2324AB & 11410 & 2007.688 & $\mathrm{~W}$ & 20 & 2.393 & 0.035 & $148.5^{*}$ & 0.3 & & & & \\
\hline $18314+0628$ & STF2329 & 11420 & 2007.688 & $\mathrm{~W}$ & 20 & 4.283 & 0.021 & $43.2^{*}$ & 0.4 & & & & \\
\hline $18320+0647$ & STT 354 & 11432 & 2007.723 & $\mathrm{R}$ & 20 & 0.592 & 0.008 & 209.0 & 1.2 & & & & \\
\hline $18360+1144$ & STT 357 & 11484 & 2007.693 & $\mathrm{~W}$ & 10 & 0.386 & 0.005 & 78.3 & 1.4 & Diff. & Val1981d & 0.03 & 1.0 \\
\hline$"$ & $"$ & $"$ & 2007.698 & $\mathrm{~W}$ & 10 & 0.389 & 0.008 & 78.2 & 1.5 & & Val1981d & 0.03 & 0.9 \\
\hline$"$ & $"$ & $"$ & 2007.701 & $\mathrm{~W}$ & 10 & 0.375 & 0.004 & 78.7 & 0.6 & & Val1981d & 0.02 & 1.4 \\
\hline $18384+0850$ & HU 198 & 11524 & 2007.690 & $\mathrm{R}$ & 20 & 0.483 & 0.013 & 128.1 & 0.4 & & Nov2007d & 0.02 & -2.4 \\
\hline $18384+3603$ & STF2362 & 11534 & 2007.690 & $\mathrm{R}$ & 20 & 4.317 & 0.036 & $186.6^{*}$ & 0.3 & & & & \\
\hline $18389+5221$ & STF2368AB & 11558 & 2007.655 & $\mathrm{R}$ & 20 & 1.897 & 0.013 & $140.5^{*}$ & 0.3 & & & & \\
\hline $18413+3018$ & STF2367AB & 11579 & 2007.769 & $\mathrm{R}$ & 10 & 0.382 & 0.004 & $75.8^{*}$ & ${ }^{k} 1.1$ & & $\mathrm{Pbx} 2000 \mathrm{~b}$ & -0.00 & 0.5 \\
\hline $18443+3940$ & STF2382AB & 11635 & 2007.728 & $\mathrm{R}$ & 20 & 2.363 & 0.015 & $348.7^{*}$ & 0.5 & & WSI2004b & -0.04 & 0.5 \\
\hline$"$ & $"$ & $"$ & $\|$ & $"$ & $"$ & $"$ & $"$ & $"$ & $"$ & & Nov2006e & -0.00 & 0.4 \\
\hline$"$ & $"$ & $"$ & 2007.778 & $\mathrm{R}$ & 20 & 2.383 & 0.017 & $348.6^{*}$ & 0.3 & & WSI2004b & -0.02 & 0.4 \\
\hline$"$ & $"$ & $"$ & $"$ & $"$ & $"$ & $"$ & $"$ & $"$ & $"$ & & Nov2006e & 0.02 & 0.3 \\
\hline $18443+3940$ & STF2383Cc-D & 11635 & 2007.728 & $\mathrm{R}$ & 20 & 2.399 & 0.023 & $78.9^{*}$ & 0.3 & & Doc1984b & 0.04 & -0.3 \\
\hline$"$ & $"$ & $"$ & 2007.775 & $\mathrm{R}$ & 20 & 2.398 & 0.014 & $79.0^{*}$ & 0.9 & & Doc1984b & 0.04 & -0.2 \\
\hline $18455+0530$ & STF2375AB & 11640 & 2007.655 & $\mathrm{R}$ & 20 & 2.540 & 0.013 & $119.0^{*}$ & 0.3 & & & & \\
\hline $18458+3431$ & STF2390 & 11669 & 2007.690 & $\mathrm{R}$ & 20 & 4.195 & 0.029 & $155.2^{*}$ & 0.3 & & & & \\
\hline $18469+5920$ & STF2410 & 11697 & 2007.693 & $\mathrm{R}$ & 20 & 1.678 & 0.016 & 86.1 & 0.7 & & & & \\
\hline $18472+3125$ & STF2397 & 11685 & 2007.693 & $\mathrm{~W}$ & 20 & 3.883 & 0.028 & $266.9^{*}$ & 0.3 & Diff. & & & \\
\hline $18540+3723$ & BU 137AB & 11811 & 2007.698 & $\mathrm{R}$ & 20 & 1.571 & 0.011 & 162.4 & 0.5 & & & & \\
\hline $18545+2037$ & STF2415 & 11816 & 2007.690 & $\mathrm{R}$ & 20 & 1.972 & 0.020 & $289.7^{*}$ & 0.5 & & & & \\
\hline $18549+3358$ & STT $525 \mathrm{AB}$ & 11834 & 2007.698 & $\mathrm{R}$ & 20 & 1.791 & 0.016 & $129.8^{*}$ & 0.3 & Diff. & & & \\
\hline $18559+0323$ & A 2193 & 11844 & 2007.698 & $\mathrm{~W}$ & 20 & 0.884 & 0.014 & 354.3 & 0.6 & & & & \\
\hline $18571+2606$ & STF2422 & 11869 & 2007.668 & $\mathrm{R}$ & 20 & 0.752 & 0.008 & 69.6 & 0.7 & & & & \\
\hline $18576+3209$ & A 260 & 11879 & 2007.698 & $\mathrm{~W}$ & 20 & 0.907 & 0.009 & 244.5 & 0.7 & & & & \\
\hline $18581+4711$ & AG 366 & 11899 & 2007.698 & $\mathrm{R}$ & 20 & 1.494 & 0.015 & 190.8 & 0.4 & & & & \\
\hline $18588+0207$ & MIL 6 & 11891 & 2007.701 & $\mathrm{~W}$ & 20 & 0.546 & 0.017 & 86.2 & 0.6 & & & & \\
\hline $18594+2936$ & STF2430 & 11914 & 2007.699 & $\mathrm{~W}$ & 20 & 1.544 & 0.012 & $186.7^{*}$ & 0.3 & & & & \\
\hline $19019+1910$ & STF2437 & 11956 & 2007.668 & $\mathrm{R}$ & 20 & 0.592 & 0.014 & 11.2 & 1.6 & & Sca2008c & 0.02 & 0.8 \\
\hline $19037+3545$ & STF2448 & 12002 & 2007.701 & $\mathrm{R}$ & 20 & 2.466 & 0.017 & 190.6 & 0.3 & & & & \\
\hline $19042+3245$ & BRD 4 & 12008 & 2007.701 & $\mathrm{~W}$ & 20 & 2.535 & 0.013 & $310.6^{*}$ & 0.3 & & & & \\
\hline $19055+3352$ & HU 940 & 12033 & 2007.701 & $\mathrm{R}$ & 20 & 0.533 & 0.035 & 196.1 & 2.3 & & Hei2001 & 0.04 & 3.8 \\
\hline $19070+1104$ & HEI 568 & - & 2007.723 & $\mathrm{R}$ & 10 & 0.301 & 0.004 & $273.0^{*}$ & 0.3 & & & & \\
\hline $19078+3856$ & STF2469AB & 12075 & 2007.723 & $\mathrm{R}$ & 20 & 1.262 & 0.022 & $127.2^{*}$ & 0.5 & & & & \\
\hline $19079+2948$ & STF2466AB & 12071 & 2007.723 & $\mathrm{~W}$ & 20 & 2.384 & 0.012 & $102.6^{*}$ & 0.6 & & & & \\
\hline $19126+1651$ & BU 139AB & 12160 & 2007.655 & $\mathrm{R}$ & 20 & 0.649 & 0.014 & 134.3 & 0.6 & & & & \\
\hline $19159+2727$ & $\mathrm{STT} 371 \mathrm{AB}$ & 12239 & 2007.688 & $\mathrm{R}$ & 20 & 0.889 & 0.011 & $160.1^{*}$ & 0.4 & & & & \\
\hline $19160+1610$ & $\mathrm{STT} 368 \mathrm{AB}$ & 12236 & 2007.723 & $\mathrm{R}$ & 20 & 1.146 & 0.009 & 218.7 & 0.8 & & & & \\
\hline $19169+6312$ & STF2509 & 12296 & 2007.688 & $\mathrm{R}$ & 20 & 1.798 & 0.009 & $328.7^{*}$ & 0.3 & & & & \\
\hline $19177+2302$ & BU 248AB & 12287 & 2007.723 & $\mathrm{R}$ & 20 & 1.703 & 0.018 & $127.8^{*}$ & 0.4 & Diff. & & & \\
\hline $19185+0105$ & STF2492AB & 12289 & 2007.731 & $\mathrm{R}$ & 20 & 3.184 & 0.038 & $2.6^{*}$ & 0.3 & Diff. & & & \\
\hline $19186+2157$ & STF2499 & 12298 & 2007.731 & $\mathrm{~W}$ & 20 & 2.579 & 0.013 & $323.5^{*}$ & 0.3 & & & & \\
\hline $19186+5358$ & A 1393 & 12315 & 2007.731 & $\mathrm{~W}$ & 20 & 0.719 & 0.009 & $253.5^{*}$ & 0.3 & & & & \\
\hline $19202+3411$ & HU 1300 & 12334 & 2007.731 & $\mathrm{~W}$ & 20 & 0.732 & 0.008 & $183.2^{*}$ & 0.8 & & & & \\
\hline $19252+0227$ & STF2513 & 12414 & 2007.731 & $\mathrm{~W}$ & 20 & 2.008 & 0.011 & $328.4^{*}$ & 0.6 & & & & \\
\hline
\end{tabular}


Table 1 - continued

\begin{tabular}{|c|c|c|c|c|c|c|c|c|c|c|c|c|c|}
\hline WDS & Name & ADS & Epoch & Fil. & $\begin{array}{l}\text { Eyep. } \\
(\mathrm{mm})\end{array}$ & $\begin{array}{c}\rho \\
(")\end{array}$ & $\begin{array}{l}\sigma_{\rho} \\
(")\end{array}$ & $\begin{array}{l}\theta \\
\left({ }^{\circ}\right)\end{array}$ & $\begin{array}{l}\sigma_{\theta} \\
\left(^{\circ}\right)\end{array}$ & Notes & Orbit & $\begin{array}{r}\Delta \rho(\mathrm{O}-\mathrm{C}) \\
(")\end{array}$ & $\begin{array}{r}\Delta \theta(\mathrm{O}-\mathrm{C}) \\
\left({ }^{\circ}\right)\end{array}$ \\
\hline $19252+3708$ & HJ 1395AB & 12427 & 2007.731 & $\mathrm{~W}$ & 20 & 2.832 & 0.014 & 63.3 & 0.3 & & & & \\
\hline $19266+2719$ & STF 2525 & 12447 & 2007.789 & $\mathrm{R}$ & 20 & 2.151 & 0.025 & $289.6^{*}$ & 0.5 & & Hei1984b & 0.06 & -0.6 \\
\hline $19269+1204$ & A 1181 & 12452 & 2007.699 & $\mathrm{R}$ & 20 & 0.672 & 0.008 & $199.1^{*}$ & 0.7 & & & & \\
\hline $19307+2758$ & MCA 55Aa,Ac & 12540 & 2007.770 & $\mathrm{R}$ & 10 & 0.350 & 0.006 & $103.3^{*}$ & 0.4 & Diff. & Sca2008a & -0.03 & -1.5 \\
\hline $19334+6203$ & STF2553 & 12626 & 2007.731 & $\mathrm{~W}$ & 20 & 0.986 & 0.009 & $127.7^{*}$ & 0.3 & & & & \\
\hline $19346+1808$ & STT 375 & 12623 & 2007.701 & $\mathrm{R}$ & 20 & 0.625 & 0.015 & 183.0 & 1.0 & & & & \\
\hline $19389+5150$ & BU 656 & 12758 & 2007.701 & $\mathrm{R}$ & 20 & 0.916 & 0.032 & 270.4 & 1.2 & & & & \\
\hline $19406+6240$ & STF2574 & 12803 & 2007.731 & $\mathrm{R}$ & 20 & 0.492 & 0.013 & 88.4 & 0.6 & & & & \\
\hline $19411+1349$ & KUI 93 & - & 2007.728 & $\mathrm{R}$ & 10 & 0.168 & 0.011 & 314.1 & 1.1 & & Doc2007d & -0.01 & -1.4 \\
\hline $19426+1150$ & STT $380 \mathrm{AB}$ & 12808 & 2007.753 & $\mathrm{R}$ & 10 & 0.403 & 0.003 & $76.8^{*}$ & 0.7 & & & & \\
\hline $19429+4043$ & STT $383 \mathrm{AB}$ & 12831 & 2007.701 & $\mathrm{R}$ & 20 & 0.822 & 0.011 & 15.7 & 0.5 & & & & \\
\hline $19438+3819$ & $\mathrm{STT} 384 \mathrm{AB}$ & 12851 & 2007.753 & $\mathrm{R}$ & 20 & 1.038 & 0.008 & $196.4^{*}$ & 0.5 & & & & \\
\hline $19450+4508$ & STF2579AB & 12880 & 2007.688 & $\mathrm{R}$ & 20 & 2.646 & 0.013 & $220.2^{*}$ & 0.3 & & Sca1983a & -0.01 & -0.8 \\
\hline $19456+3337$ & STF2576AB & 12889 & 2007.753 & $\mathrm{R}$ & 20 & 2.871 & 0.014 & $160.3^{*}$ & 0.4 & & Sod1999 & 0.00 & 0.1 \\
\hline $19458+4033$ & STT 385 & 12904 & 2007.753 & $\mathrm{R}$ & 20 & 1.242 & 0.014 & $50.4^{*}$ & 0.9 & & & & \\
\hline $19471+3321$ & HU 758 & 12930 & 2007.723 & $\mathrm{~W}$ & 20 & 0.896 & 0.026 & 144.8 & 1.4 & & & & \\
\hline $19479+2414$ & DA 10 & 12948 & 2007.753 & $\mathrm{R}$ & 20 & 0.667 & 0.011 & $307.0^{*}$ & 0.4 & & & & \\
\hline $19482+7016$ & STF2603 & 13007 & 2007.770 & $\mathrm{R}$ & 20 & 3.141 & 0.016 & $19.6^{*}$ & 0.3 & & & & \\
\hline $19484+2212$ & STF2584 & 12957 & 2007.723 & W & 20 & 1.906 & 0.010 & 293.6 & 0.4 & & & & \\
\hline $19486+2458$ & STF2586AB & 12964 & 2007.753 & $\mathrm{~W}$ & 20 & 3.759 & 0.028 & $226.0^{*}$ & 0.3 & Diff. & & & \\
\hline $19487+1149$ & STF2583AB & 12962 & 2007.669 & $\mathrm{R}$ & 20 & 1.432 & 0.008 & $105.1^{*}$ & 0.3 & & & & \\
\hline $19487+3519$ & STT 387 & 12972 & 2007.778 & $\mathrm{R}$ & 10 & 0.553 & 0.004 & $125.6^{*}$ & 0.6 & & WSI2006b & -0.01 & -2.0 \\
\hline $19515+2332$ & BU 978 & 13030 & 2007.770 & $\mathrm{~W}$ & 20 & 1.032 & 0.011 & $58.3^{*}$ & 0.7 & & & & \\
\hline $19524+2551$ & $\mathrm{STT} 388 \mathrm{AB}$ & 13050 & 2007.778 & $\mathrm{R}$ & 20 & 3.873 & 0.019 & $137.2^{*}$ & 0.3 & & & & \\
\hline $19525+2227$ & HO 580 & 13055 & 2007.770 & $\mathrm{R}$ & 20 & 0.699 & 0.017 & $274.8^{*}$ & 2.2 & & & & \\
\hline $19535+2405$ & DJU 4 & - & 2007.770 & $\mathrm{R}$ & 20 & 1.336 & 0.016 & $245.8^{*}$ & 0.3 & & Cve2007c & 0.02 & -0.5 \\
\hline $19540+1518$ & STF2596 & 13082 & 2007.669 & $\mathrm{R}$ & 20 & 2.056 & 0.020 & 299.6 & 0.3 & & & & \\
\hline $19556+5226$ & STF2605AB & 13148 & 2007.775 & $\mathrm{R}$ & 20 & 2.898 & 0.041 & $175.1^{*}$ & 0.4 & Diff. & & & \\
\hline $19576+1524$ & A $1663 \mathrm{AB}$ & 13166 & 2007.778 & W & 20 & 1.293 & 0.008 & $236.6^{*}$ & 0.3 & & & & \\
\hline $19579+4216$ & STF2607AB-C & 13186 & 2007.778 & $\mathrm{R}$ & 20 & 2.998 & 0.015 & $288.6^{*}$ & 0.3 & Diff. & & & \\
\hline $19584-0214$ & $\mathrm{AC} 12$ & 13178 & 2007.778 & $\mathrm{R}$ & 20 & 1.458 & 0.013 & $298.8^{*}$ & 0.7 & & & & \\
\hline $19585+3317$ & STF2606AB & 13196 & 2007.775 & W & 20 & 0.728 & 0.009 & $144.8^{*}$ & 0.7 & & & & \\
\hline $19586+3806$ & STF2609 & 13198 & 2007.775 & $\mathrm{R}$ & 20 & 1.954 & 0.015 & $22.0^{*}$ & 0.4 & & & & \\
\hline $19591+3532$ & STF2610AB & 13204 & 2007.783 & W & 20 & 4.272 & 0.021 & $295.5^{*}$ & 0.3 & & & & \\
\hline $19594+3206$ & A 378 & 13212 & 2007.778 & $\mathrm{~W}$ & 20 & 0.887 & 0.008 & $293.6^{*}$ & 0.5 & & & & \\
\hline $20010+3742$ & BU $1289 \mathrm{AB}$ & 13262 & 2007.783 & W & 20 & 0.633 & 0.030 & $54.8^{*}$ & 0.9 & & & & \\
\hline $20017-0012$ & H $193 \mathrm{AB}$ & 13259 & 2007.783 & W & 20 & 1.827 & 0.022 & $297.7^{*}$ & 0.5 & & & & \\
\hline $20020+2456$ & STT 395 & 13277 & 2007.729 & $\mathrm{R}$ & 20 & 0.802 & 0.008 & 123.8 & 0.3 & & & & \\
\hline $20027+2939$ & COU1473 & - & 2007.728 & $\mathrm{~W}$ & 20 & 0.559 & 0.008 & 347.2 & 0.9 & & & & \\
\hline $20028+1435$ & STF2616 & 13290 & 2007.783 & W & 20 & 3.548 & 0.044 & $266.8^{*}$ & 0.3 & Diff. & & & \\
\hline $20035+3601$ & STF2624AB & 13312 & 2007.775 & $\mathrm{R}$ & 20 & 1.945 & 0.021 & $174.2^{*}$ & 0.3 & & & & \\
\hline $20040+1221$ & A 1194 & 13314 & 2007.789 & W & 20 & 1.000 & 0.025 & $315.4^{*}$ & 0.3 & & & & \\
\hline $20043+3033$ & STF2626 & 13329 & 2007.789 & $\mathrm{R}$ & 20 & 1.013 & 0.035 & $126.5^{*}$ & 1.5 & & & & \\
\hline $20051-0418$ & BU 56 & 13334 & 2007.789 & $\mathrm{R}$ & 20 & 1.355 & 0.008 & $186.6^{*}$ & 0.7 & & & & \\
\hline $20056+6342$ & STF2642AB & 13392 & 2007.789 & $\mathrm{R}$ & 20 & 1.710 & 0.023 & 185.6 & 0.6 & & & & \\
\hline $20074+3543$ & STT 398AB & 13405 & 2007.843 & $\mathrm{R}$ & 20 & 0.952 & 0.009 & $82.7^{*}$ & 0.7 & & & & \\
\hline $20080+4223$ & A 382 & 13415 & 2007.843 & $\mathrm{R}$ & 20 & 1.678 & 0.008 & $94.7^{*}$ & 0.3 & Diff. & & & \\
\hline $20137+2414$ & STF2653 & 13543 & 2007.843 & $\mathrm{R}$ & 20 & 2.878 & 0.017 & $274.4^{*}$ & 0.4 & Diff. & & & \\
\hline $20144+4206$ & $\mathrm{STT} 403 \mathrm{AB}$ & 13572 & 2007.723 & $\mathrm{R}$ & 20 & 0.951 & 0.015 & $170.6^{*}$ & 0.5 & & & & \\
\hline $20153+2536$ & BU 983AB & 13589 & 2007.843 & $\mathrm{R}$ & 10 & 0.467 & 0.005 & $183.8^{*}$ & 0.4 & Diff. & & & \\
\hline $20181+4044$ & STF2666AB & 13672 & 2007.854 & $\mathrm{R}$ & 20 & 2.758 & 0.014 & $244.9^{*}$ & 0.3 & & & & \\
\hline $20184+5524$ & STF2671AB & 13692 & 2007.854 & $\mathrm{R}$ & 20 & 3.713 & 0.019 & $336.8^{*}$ & 0.3 & & & & \\
\hline $20198+4522$ & STT 406 & 13723 & 2007.854 & $\mathrm{R}$ & 20 & 0.356 & 0.008 & $101.1^{*}$ & 0.3 & & Hei1976 & -0.08 & -3.9 \\
\hline $20303+1054$ & $\mathrm{BU} 63 \mathrm{AB}$ & 13920 & 2007.688 & $\mathrm{R}$ & 20 & 0.916 & 0.008 & $348.5^{*}$ & 0.4 & & & & \\
\hline
\end{tabular}


Table 1 - continued

\begin{tabular}{|c|c|c|c|c|c|c|c|c|c|c|c|c|}
\hline WDS & Name & ADS & Epoch & Fil. & $\begin{array}{l}\text { Eyep. } \\
(\mathrm{mm})\end{array}$ & $\begin{array}{c}\rho \\
(")\end{array}$ & $\begin{array}{l}\sigma_{\rho} \\
(")\end{array}$ & $\begin{array}{ll}\theta & \sigma_{\theta} \\
\left({ }^{\circ}\right) & \left(^{\circ}\right)\end{array}$ & Notes & Orbit & $\begin{array}{r}\Delta \rho(\mathrm{O}-\mathrm{C}) \\
(")\end{array}$ & $\begin{array}{r}\Delta \theta(\mathrm{O}-\mathrm{C}) \\
\left({ }^{\circ}\right)\end{array}$ \\
\hline $20318+4933$ & BU 1136 & 13976 & 2007.753 & $\mathrm{R}$ & 20 & 0.575 & 0.015 & $215.2^{*} 0.5$ & & & & \\
\hline $20335+0527$ & STF2696AB & 13997 & 2007.778 & W & 20 & 0.546 & 0.014 & 297.91 .1 & & & & \\
\hline $20356+3510$ & STF2702 & 14045 & 2007.723 & W & 20 & 3.204 & 0.016 & $205.3^{*} 0.3$ & & & & \\
\hline $20377+3322$ & STF2705AB & 14078 & 2007.906 & $\mathrm{R}$ & 20 & 3.172 & 0.016 & $261.6^{*} 0.3$ & & & & \\
\hline $20396+4035$ & STT $410 \mathrm{AB}$ & 14126 & 2007.775 & $\mathrm{R}$ & 20 & 0.843 & 0.013 & $\begin{array}{ll}6.1 & 0.5\end{array}$ & & & & \\
\hline $20423+5723$ & BU 152 & 14196 & 2007.843 & $\mathrm{R}$ & 20 & 1.159 & 0.022 & $82.3^{*} 1.3$ & & & & \\
\hline $20450+1244$ & $\mathrm{BU} 64 \mathrm{AB}$ & 14238 & 2007.844 & $\mathrm{R}$ & 20 & 0.651 & 0.008 & $\begin{array}{ll}351.7 & 0.3\end{array}$ & & Bdl2007a & -0.01 & -1.1 \\
\hline $20511+5125$ & BU 155AB & 14370 & 2007.770 & $\mathrm{R}$ & 20 & 0.677 & 0.026 & 36.31 .4 & & & & \\
\hline $20541+4507$ & STT 422 & 14411 & 2007.854 & $\mathrm{~W}$ & 20 & 2.587 & 0.014 & $332.0^{*} 0.3$ & & & & \\
\hline $20548+3242$ & STT 418 & 14421 & 2007.789 & $\mathrm{R}$ & 20 & 0.977 & 0.019 & $285.0 \quad 0.7$ & & & & \\
\hline $20585+5028$ & STF2741AB & 14504 & 2007.789 & $\mathrm{R}$ & 20 & 1.966 & 0.010 & $25.5^{*} 0.3$ & & & & \\
\hline $20593+1534$ & STT $424 \mathrm{AB}$ & 14505 & 2007.844 & $\mathrm{R}$ & 20 & 0.511 & 0.008 & $305.9 \quad 0.4$ & & & & \\
\hline $20598+2004$ & STF2739 & 14515 & 2007.914 & W & 20 & 3.331 & 0.017 & $252.3^{*} 0.3$ & Elon. & & & \\
\hline $21008+4635$ & BU 156 & 14561 & 2007.778 & W & 20 & 1.025 & 0.012 & $235.0^{*} 0.4$ & & & & \\
\hline $21018+3916$ & STF2746 & 14558 & 2007.778 & $\mathrm{R}$ & 20 & 1.201 & 0.020 & $320.6^{*} 0.7$ & & & & \\
\hline $21031+0132$ & STF2744AB & 14573 & 2007.844 & $\mathrm{R}$ & 20 & 1.273 & 0.015 & $114.3^{*} 0.4$ & & Pop1969b & 0.06 & 5.6 \\
\hline $21093+3131$ & COU1333 & - & 2007.770 & W & 20 & 0.628 & 0.017 & $255.2 \quad 1.2$ & & & & \\
\hline $21112+5620$ & DOO 16 & 14739 & 2007.789 & W & 20 & 1.102 & 0.008 & $28.5^{*} 0.8$ & & & & \\
\hline $21143+4109$ & STT 432 & 14778 & 2007.914 & $\mathrm{R}$ & 20 & 1.333 & 0.010 & $114.7^{*} 0.3$ & & & & \\
\hline $21197+0931$ & STF2786 & 14856 & 2007.950 & $\mathrm{R}$ & 20 & 2.738 & 0.014 & $188.4^{*} 0.3$ & & & & \\
\hline $21208+3227$ & STT $437 \mathrm{AB}$ & 14889 & 2007.950 & $\mathrm{R}$ & 20 & 2.402 & 0.012 & $20.6^{*} 0.6$ & & & & \\
\hline $21252+3129$ & A 1220 & 14957 & 2007.950 & W & 20 & 1.624 & 0.019 & $157.5^{*} 0.7$ & & & & \\
\hline $21280+4305$ & HO 160 & 15000 & 2007.950 & W & 20 & 2.036 & 0.010 & $182.5^{*} 0.5$ & & & & \\
\hline $21330+2043$ & STF2804AB & 15076 & 2007.958 & $\mathrm{R}$ & 20 & 3.291 & 0.050 & $356.8^{*} 0.8$ & & & & \\
\hline $21521+2748$ & HO 171 & 15401 & 2007.914 & W & 20 & 0.750 & 0.014 & $\begin{array}{ll}341.9 & 0.9\end{array}$ & Elon. & & & \\
\hline $21555+5232$ & STT 456AB & 15460 & 2007.958 & $\mathrm{R}$ & 20 & 1.615 & 0.012 & $36.3^{*} 0.3$ & & & & \\
\hline $22009+4338$ & HO $175 \mathrm{AB}$ & 15547 & 2007.844 & $\mathrm{R}$ & 20 & 0.868 & 0.028 & $318.9^{*} 0.7$ & & & & \\
\hline $22029+4439$ & BU 694AB & 15578 & 2007.844 & $\mathrm{R}$ & 20 & 1.000 & 0.008 & $6.1^{*} 0.3$ & & & & \\
\hline $22044+1339$ & STF2854 & 15596 & 2007.966 & $\mathrm{R}$ & 20 & 1.691 & 0.008 & $82.7^{*} 0.5$ & & & & \\
\hline $22071+0034$ & STF2862 & 15639 & 2007.966 & $\mathrm{R}$ & 20 & 2.498 & 0.012 & $95.4^{*} 0.3$ & & & & \\
\hline $22110+2429$ & EGG 4 & - & 2007.844 & W & 20 & 0.563 & 0.008 & $149.6^{*} 0.3$ & & & & \\
\hline $22110+6324$ & STF2879AB & 15712 & 2007.958 & $\mathrm{R}$ & 20 & 0.776 & 0.013 & 232.31 .0 & & & & \\
\hline $22117+5743$ & A $625 \mathrm{AB}$ & 15726 & 2007.969 & $\mathrm{R}$ & 20 & 0.547 & 0.018 & $80.1 \quad 1.2$ & & & & \\
\hline $22126+3013$ & HO 179AB & 15738 & 2007.879 & $\mathrm{~W}$ & 20 & 0.899 & 0.008 & $280.0^{*} 0.7$ & & & & \\
\hline $22128+1355$ & HU 978 & 15735 & 2007.879 & W & 20 & 1.129 & 0.011 & $206.1^{*} 0.3$ & & & & \\
\hline $22136+5234$ & BU 991 & 15756 & 2007.964 & W & 20 & 0.657 & 0.008 & $137.8^{*} 0.5$ & & & & \\
\hline $22145+0759$ & STF2878AB & 15767 & 2007.966 & $\mathrm{R}$ & 20 & 1.495 & 0.008 & $115.1^{*} 0.3$ & & & & \\
\hline $22146+2934$ & STF2881 & 15769 & 2007.915 & $\mathrm{R}$ & 20 & 1.311 & 0.011 & $75.8^{*} 0.3$ & & & & \\
\hline $22158+4354$ & HO $180 \mathrm{AB}$ & 15794 & 2007.915 & W & 20 & 0.758 & 0.008 & $239.5^{*} 1.0$ & & & & \\
\hline $22202+2931$ & BU 1216 & 15843 & 2007.969 & $\mathrm{~W}$ & 20 & 0.887 & 0.011 & $278.0^{*} 0.3$ & & & & \\
\hline $22206+5349$ & BU 379 & 15856 & 2007.950 & W & 20 & 1.107 & 0.014 & $335.1^{*} 0.5$ & & & & \\
\hline $22269+6343$ & KR 59 & 15954 & 2007.950 & $\mathrm{~W}$ & 20 & 1.752 & 0.009 & $344.9 * 0.4$ & & & & \\
\hline $22288-0001$ & STF2909AB & 15971 & 2007.966 & $\mathrm{R}$ & 20 & 2.042 & 0.010 & $170.8^{*} 0.3$ & & Ole2004a & -0.13 & -5.2 \\
\hline$"$ & $"$ & $"$ & $"$ & $"$ & $"$ & $"$ & $"$ & $" \quad "$ & & This paper & -0.03 & -1.9 \\
\hline $22302+2228$ & HU 388 & 15992 & 2007.966 & $\mathrm{R}$ & 20 & 0.494 & 0.008 & $57.1^{*} 0.9$ & & Doc2008c & -0.02 & -1.6 \\
\hline $22305+6137$ & HU 981 & 16011 & 2007.964 & $\mathrm{~W}$ & 10 & 0.298 & 0.003 & $215.3^{*} 0.9$ & & & & \\
\hline $22345+4046$ & COU1838Aa,Ab & - & 2007.969 & $\mathrm{~W}$ & 20 & 1.097 & 0.019 & $164.8^{*} 0.9$ & Diff. & & & \\
\hline $22426+4401$ & A $414 \mathrm{AB}$ & 16204 & 2007.882 & W & 20 & 1.706 & 0.009 & $194.1^{*} 0.3$ & & & & \\
\hline $22431+4710$ & STT 476A-BC & 16214 & 2007.967 & $\mathrm{R}$ & 20 & 0.488 & 0.008 & $301.1^{*} 0.7$ & & & & \\
\hline $22470+4446$ & A $189 \mathrm{AB}$ & 16266 & 2007.967 & W & 20 & 0.991 & 0.008 & $26.0^{*} 0.6$ & & & & \\
\hline $22496+6633$ & STF2948 & 16298 & 2007.972 & $\mathrm{R}$ & 20 & 2.685 & 0.022 & $4.4^{*} 0.5$ & & & & \\
\hline $22507+5107$ & HLD 54 & 16307 & 2007.972 & $\mathrm{R}$ & 20 & 1.874 & 0.012 & $17.1^{*} 0.8$ & & & & \\
\hline $22514+2623$ & HO $482 \mathrm{AB}$ & 16314 & 2007.972 & $\mathrm{R}$ & 20 & 0.489 & 0.016 & $18.5^{*} 1.3$ & & Sta1982b & -0.00 & 2.1 \\
\hline $22520+5743$ & A 632 & 16326 & 2007.972 & $\mathrm{R}$ & 20 & 0.511 & 0.008 & $137.9^{*} 0.7$ & & Hei1991 & 0.02 & -3.2 \\
\hline $22564+2257$ & COU 240 & - & 2007.844 & $\mathrm{R}$ & 20 & 0.766 & 0.013 & 291.11 .2 & & & & \\
\hline
\end{tabular}



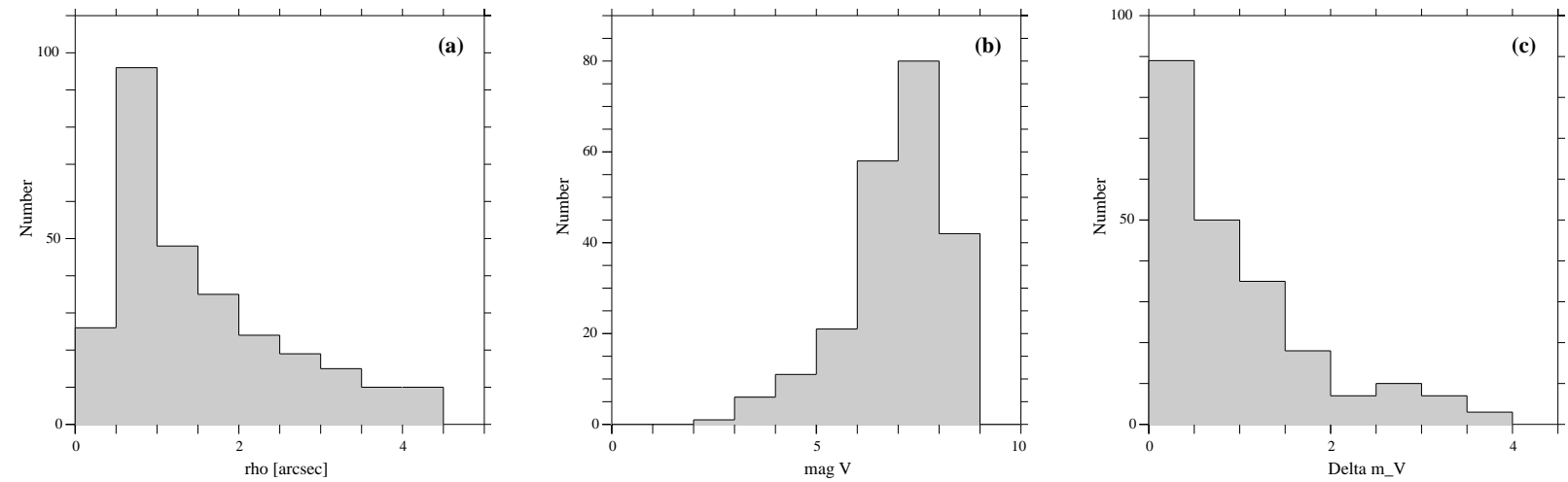

Fig. 1 Distribution of the angular separations of the 283 measurements of Table 1 (a), of the visual magnitudes of the corresponding objects (b) and of the differences of magnitude between the two components of those binaries (c).

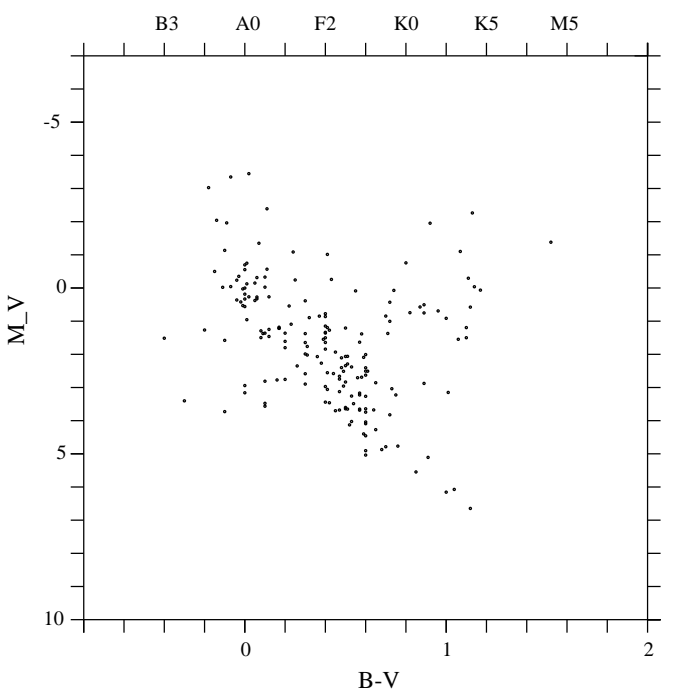

Fig. 2 HR diagram of the binaries measured in Table 1, for which Hipparcos parallaxes were obtained with a relative error smaller than $50 \%$ (184 objects).

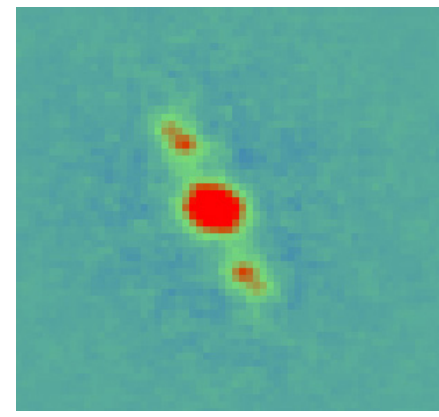

Fig. 3 Auto-correlation of the triple star ADS 11344.

were made in $V$, whereas we observed with no filter, which corresponds to a bandpass close to that of the $R$ filter (see Sect. 2). There can exist a quadrant inversion between $V$ and $R$, since the spectral type of the primary component is A0 and is probably associated to a cooler companion.
ADS 10905: Our quadrant determination $Q=2$ in $R$ is consistent with the values we found in 2004.690 in Merate (Paper II) and in 1998.654 at the Pic du Midi with a 2m-telescope (Scardia et al., 2000a). It is however in disagreement with the quadrant $\mathrm{Q}=4$ reported for the other 58 observations listed in IC4, made in $V$. Like ADS 10, this discrepancy can be due to a quadrant inversion that is likely since $\Delta m_{V}=0.12$, with a spectral class of A0 III for the primary.

ADS 11558: Our value of $\mathrm{Q}=2$ in $R$ is inconsistent with the fourth quadrant given for the 27 measures listed in IC4, all made in $V$. The Wilson spectral classes are A1n, for the primary and G0 for the secondary (WDS Catalogue notes). The small difference in magnitude, $\Delta m_{V}=0.14$, might account for the quadrant inversion since the G0 component could appear as the brightest one in $R$. We already observed this object in 2004.657 (Paper II) but the seeing conditions were not good enough for enabling the quadrant determination.

ADS 15954: Our quadrant $Q=4$ obtained with no filter is in agreement with Tycho measurement of 1991.69 (Høg et al. 2000), and in disagreement with the other 9 observations reported in IC4, made in $V$. For this object, the components have a larger brightness difference, with $\Delta m_{V}=0.4$, and the spectral type of the primary is $09 \mathrm{~V}$.

ADS 16204: Here also, our value of $Q=3$ obtained with no filter is in agreement with Tycho measurement of 1991.76 (Høg et al. 2000) and in disagreement with the other 7 observations reported in IC4, all made in $V$. The very small value of $\Delta m_{V}=0.06$ well explains the difficulty of determining the good quadrant. Furthermore, we noticed an inversion in the magnitudes of the components tabulated in the WDS Catalogue (the faintest magnitude being placed in the column of the primary component and vice-versa), which might be another source of error. We observed this binary also on 2006.951 (Paper VI) but the low signal-to-noise ratio did not allow us to determine the quadrant. 
Table 1 - continued

\begin{tabular}{|c|c|c|c|c|c|c|c|c|c|c|c|c|}
\hline WDS & Name & ADS & Epoch & Fil. & $\begin{array}{l}\text { Eyep. } \\
(\mathrm{mm})\end{array}$ & $\begin{array}{c}\rho \\
(")\end{array}$ & $\begin{array}{l}\sigma_{\rho} \\
(")\end{array}$ & $\begin{array}{ll}\theta & \sigma_{\theta} \\
\left(^{\circ}\right) & \left(^{\circ}\right)\end{array}$ & Notes & Orbit & $\begin{array}{r}\Delta \rho(\mathrm{O}-\mathrm{C}) \\
(")\end{array}$ & $\begin{array}{r}\Delta \theta(\mathrm{O}-\mathrm{C}) \\
\left({ }^{\circ}\right)\end{array}$ \\
\hline $22565+6252$ & STF2961 & 16394 & 2007.950 & $\mathrm{~W}$ & 20 & 1.870 & 0.009 & $347.5^{*} 0.3$ & & & & \\
\hline $23103+3229$ & BU 385AB & 16561 & 2007.879 & $\mathrm{R}$ & 20 & 0.657 & 0.008 & $85.8^{*} 0.8$ & & Lin2008a & -0.01 & -0.2 \\
\hline $23162+5424$ & A 1482 & 16641 & 2007.967 & $\mathrm{~W}$ & 20 & 1.315 & 0.011 & $87.7^{*} 0.4$ & & & & \\
\hline $23188+2513$ & STF3000 & 16664 & 2007.972 & W & 20 & 3.397 & 0.017 & $50.2^{*} 0.3$ & & & & \\
\hline $23420+2018$ & STT 503AB & 16937 & 2007.879 & W & 20 & 1.060 & 0.008 & $132.3^{*} 0.3$ & & & & \\
\hline $23439+0715$ & STF3033 & 16958 & 2007.972 & W & 20 & 3.006 & 0.024 & $3.2^{*} 0.6$ & & & & \\
\hline $23487+6453$ & STT 507AB & 17020 & 2007.964 & $\mathrm{R}$ & 20 & 0.705 & 0.012 & $317.1^{*} 0.4$ & & Zul1977b & -0.02 & 2.2 \\
\hline $23522+4331$ & BU 728 & 17063 & 2007 & $\mathrm{~W}$ & 20 & 1.246 & 0.008 & $8.3 \quad 0.3$ & & & & \\
\hline $23576+4804$ & A 799 & 17122 & 2007.915 & W & 20 & 2.019 & 0.010 & $190.9^{*} 0.3$ & & & & \\
\hline $23579+5723$ & STF3047AB & 17126 & 2007.964 & W & 20 & 1.122 & 0.017 & $70.5^{*} 0.8$ & & & & \\
\hline $23590+5315$ & HLD 59AB & 17141 & 2007.964 & W & 20 & 1.173 & 0.008 & $12.6^{*} 0.4$ & & & & \\
\hline $23590+5545$ & STF3049AB & 17140 & 2007.964 & $\mathrm{R}$ & 20 & 3.073 & 0.017 & $325.9^{*} 0.3$ & & & & \\
\hline $23595+3343$ & STF3050AB & 17149 & 2007.915 & $\mathrm{R}$ & 20 & 2.196 & 0.011 & $334.3^{*} 0.5$ & & Sta1977b & 0.11 & -0.1 \\
\hline \multicolumn{13}{|c|}{ 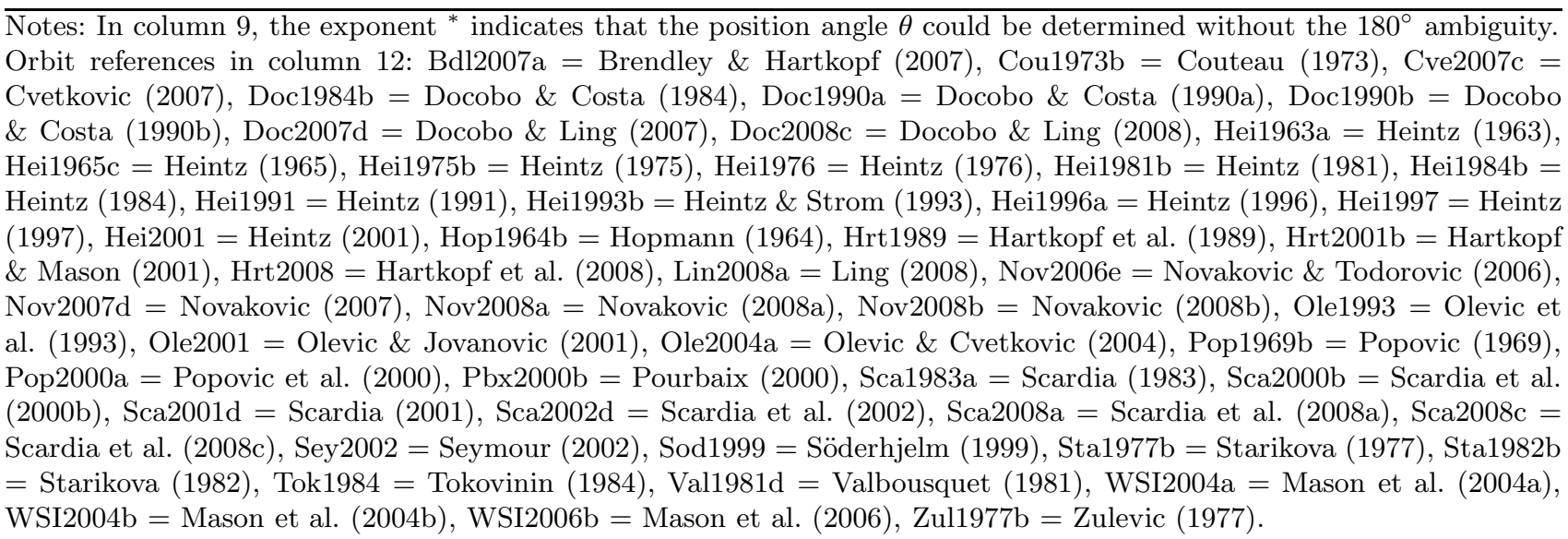 } \\
\hline
\end{tabular}

ADS 16307: Our quadrant $\mathrm{Q}=1$ determined in $R$ is in agreement with Tycho determination of 1991.72 (Høg et al. 2000), but in disagreement with the other 5 observations reported in IC4, and made in $V$. This discrepancy is difficult to explain since $\Delta m_{V}=0.81$. The primary spectral class is A0.

ADS 16958: Here conversely, our quadrant $Q=1$ determined without filter is consistent with all the observations reported in IC4 (all made in $V$ ) but not with the Tycho measurement $(\mathrm{Q}=3$ ) of 1992.07 (Høg et al. 2000) whose quadrant was adopted by the WDS Catalogue. The spectral class is F2 and the difference in magnitude of the components is significant: $\Delta m_{V}=0.33$.

ADS 17122: Our determination of $Q=3$ made with no filter is in agreement with Tycho measurements of 1991.67 (Høg et al. 2000), but not with the first quadrant (adopted by the WDS Catalogue) of the other 7 observations reported in IC4, all made in V. The difference in magnitude of the components is $\Delta m_{V}=0.17$ and the spectral class A5.

\subsection{Comparison with published ephemerides}

The $(O-C)$ (Observed minus Computed) residuals of the measurements for the 65 systems with a known orbit in Table 1 are displayed in Cols. 13 and 14 for the separation $\rho$ and position angle $\theta$, respectively. The orbital elements used for computing the ephemerides were retrieved from the "Sixth Catalogue of Orbits of Visual Binary Stars" (Hartkopf \& Mason, 2009, hereafter OC6). The corresponding authors are given in Col. 12, using the style of the OC6 references.

The residuals reported in this table were computed with the most recent orbits found in OC6, but for some objects (i.e., for ADS 293 and 11635), we also give the $O-C$ values relative to old orbits found in the previous issues of OC6, when they are still valid. For STF 2909 AB, we also give the residuals obtained with our new orbits presented in Sect. 4, for comparison.

For most of the orbits used for computing the residuals reported in Table 1 , the equinox of the node $\Omega$ was 2000.0 , and the correction to be applied to the computed position angle was negligible. Such a correction 


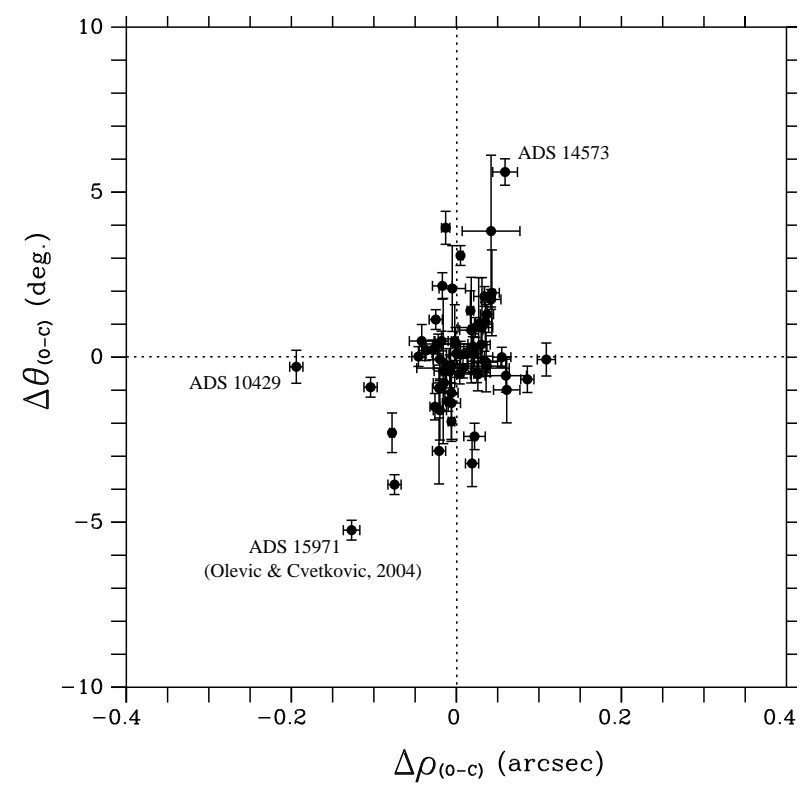

Fig. 4 Residuals of the measurements of Table 1 from the published orbits.

was only necessary for ADS 9378 (equinox: 1950.0) and for ADS 9969, 16314 and 17149 (equinox: 1900.0).

Fig. 4 shows that the residuals are well centered around the origin, with a rather large scatter that can be explained by the (old) age of many orbits. The mean values computed with the residuals of Table 1 are $<$ $\left.\Delta \rho_{O-C}\right\rangle=0^{\prime \prime} .00 \pm 0^{\prime \prime} .05$ and $\left\langle\Delta \theta_{O-C}\right\rangle=0^{\circ} .2 \pm 1^{\circ} .7$. The small values obtained for those offsets provide an additional validation of our calibration made with a grating mask (see Paper III), which is in good agreement with the measurements made by the other observers. In the following, we examine the cases of ADS 10429, 14573 and 15971 that appear with the largest residuals in Fig. 4.

ADS 10429: this couple is difficult to measure because of a large brightness difference between the two components: $\Delta m_{V}=2.5$. This may explain why there are only 3 speckle measurements in IC4: two made in 1995 by the US Naval Obs. in Washington, and one obtained by our group in 2004.659 with $\rho=0^{\prime \prime} .867 \pm 0^{\prime \prime} .006$ and $\theta=10^{\circ} .3 \pm 0^{\circ} .9$ (Paper II). All the other measurements were obtained visually. The revision of Olevic et al (1993)'s orbit would be too premature, because it is still satisfactory in terms of position angle, and also because the orbital arc covered by the companion is very small.

ADS 14573: here also the orbital arc covered by the companion is too small to justify a new computation: the resulting orbit would be completely undetermined (grade 5).

ADS 15971: the recent orbit of STF 2909 AB computed by Olevic \& Cvetkovic (2004) leads to rather large residuals with both our measurement and the last ones reported in IC4. The companion is moving closer to the primary than expected by Olevic's orbit. We propose a new orbit in the next section.

\section{Revised orbits of the multiple system Zeta Aqr - ADS 15971}

\subsection{Visual orbit AB of Zeta Aqr}

ADS 15971 - STF 2909 AB - HIP 110960 - Zeta Aqr, is a famous binary star, with more than 1200 measurements made since its discovery in 1777 , by Christian Mayer in Mannheim with a Bird mural quadrant. It was first reported in Mayer's catalogue (actually the first catalogue of binary stars) that contained 89 couples and was published in the "Astronomische Jahrbuch für 1784". The first real measures of ADS 15971 were made by W. Herschel in 1779 with a 6 -inch telescope.

This object was observed with PISCO at the Pic du Midi in 1998 and in Merate in 2004, 2005 and 2007, and two of us have also contributed to this effort with micrometer observations. MS used a 23-cm refractor in Merate in 1982 and 1984, and the 38-cm GPO in La Silla in 1992. RWA obtained an annual series of measures between 1990 and 2008 using the 20-cm refractor at Cambridge University Observatories. The whole list of measurements used for computing the new orbits was kindly provided by B. Mason from the WDS data base maintained by the United States Naval Observatory. We also added a recent measurements made with a micrometer by RWA with the 20 -cm Cambridge Observatory refractor (Argyle, 2009).

We first computed the preliminary orbital elements with the analytical method of Kowalsky (1873). We then used them as initial values for the least-squares method of Hellerich (1925), which leads to an improvement of the orbital elements with an estimation of their errors.

The total list of 1211 measurements was obtained with a wide range of telescopes (with apertures from 7 -cm to $4-\mathrm{m}$ ). To obtain a more robust solution, we rejected the aberrant measurements for which the residuals were larger than 1.7- $\sigma$, i.e., with $\Delta \rho_{O-C}>0^{\prime \prime} .31$ and $\Delta \theta_{O-C}>4^{\circ} 6$. This resulted in a selection of 1189 observations. The final orbital elements are presented in Table 2 with the errors obtained by Hellerich's method. For all the tables of this section, we use the same format as for the previous papers. A full description can be found, for instance in Paper VI. The mean standard deviations of the residuals are $0^{\prime \prime} .22$ and $2^{\circ} .9$ for $\rho$ and $\theta$, respectively. This scatter is explained by the large range of instruments and epochs, and also by the perturbation of a third invisible body (see below).

The apparent orbit is shown in Fig. 5 as a solid line and the observational data plotted as small crosses or, in the case of PISCO observations, as small circles. 


\subsection{Perturbation orbit Bb-P of Zeta Aqr}

The zoomed part of the orbit in Fig. 5b on the most recent observations exhibits an oscillatory trend which betrays the presence of a third star perturbing the orbital motion of the AB couple. This property was first noticed by Strand (1942), who published the first orbits for the close and wide pairs simultaneously. A series of subsequent studies (Strand 1942, Franz 1958, Harrington 1968, Heintz 1984c) precised the parameters of the perturbation orbit with a period of 25.5 years and a semi-major axis of $0^{\prime \prime} .06$. The mass of the third star was estimated at $0.22 \mathrm{M}_{\odot}$ by Harrington (1968) or at $0.4 \mathrm{M}_{\odot}$ by Heintz (1984c).

It is difficult to locate this companion, since the masses of the $\mathrm{A}$ and $\mathrm{B}$ components are similar and the presence of a companion "a" close to A or another companion "b" close to B would produce similar effects on the $\mathrm{AB}$ orbit. On the basis of parallax observations, Franz (1958) linked it to a possible Bb pair, whereas Heintz (1984) favoured a hypothetical Aa pair.

At optical wavelengths, Weigelt (1983) found a close companion to Zeta Aqr A with a separation of $0^{\prime \prime} .064 \pm 0.005$ in 1978.964 , which is much too small to correspond to the perturbation orbit. In near infrared, a companion was detected close to Zeta Aqr B at $0^{\prime \prime} .17$ by McCarthy et al (1982) with the Kitt Peak 3.8-m telescope. The angular separation and the estimated mass were both in good agreement with Harrington's orbit. We will assume in this paper that this observation is valid and that the perturbation is due to the $\mathrm{Bb}$ couple detected by McCarthy et al. According to the convention used by the OC6, the perturbation orbit will be called $\mathrm{Bb}-\mathrm{P}$, since its corresponds to the orbit of $\mathrm{B}$ relative to the center of mass of the outer Bb system.

Using all the photographic and speckle measurements published since 1923, we computed the residuals of our orbit of ADS 15971 AB in Cartesian coordinates, from which we derived the elements of the perturbation orbit Bb-P and its uncertainties with Hellerich (1925)'s least-squares method. To our knowledge, the uncertainties on those elements had never been computed before. The results are presented in Tables 2, 3 and in Fig. 6.

The $(O-C)$ residuals obtained with the combination of orbits $\mathrm{AB}$ and $\mathrm{Bb}-\mathrm{P}$, restricted for reasons of space to the observations made after 2002, are given in Table 4 . The corresponding mean standard deviations are $0^{\prime \prime} .11$ and $1^{\circ} .25$ for $\rho$ and $\theta$, respectively, which is about half of the values obtained with the orbit $\mathrm{AB}$ alone (see Sect. 4.1). The combined ephemerides for 2009-2020 are presented in Table 5.

Our two orbits are still temporary since only half of the $\mathrm{AB}$ orbit has been monitored yet. The orbital elements of the main orbit are still poorly known (for instance the period has an uncertainty of $10 \%$ ).

\subsection{Discussion: nature of the perturbing component}

In the SIMBAD data base, the two components $\mathrm{A}$ and B of Zeta Aqr are identified separately as HD 213052 $\left(\mathrm{F} 3 \mathrm{~V}, \mathrm{~m}_{V}=4.42\right)$ and HD $213051\left(\mathrm{~F} 6 \mathrm{IV}, \mathrm{m}_{V}=4.5\right)$. Some physical parameters derived for this system from our orbital elements are presented in Table 6. Both the linear size of $a$ (Col. 5) and $\mathfrak{M}_{\text {total }}$ (Col. 6) were computed from our orbital elements and using the Hipparcos parallax revised by van Leeuwen (2007) and reported in Col. 3. Using the relation we established for IV-type stars (Scardia et al, 2008c, p 380) we derived a total mass of $3.59 \pm 0.96 \mathrm{M}_{\odot}$. The theoretical value for two stars F3V+F6IV is $\mathfrak{M}_{\text {total }}=2.64 \mathrm{M}_{\odot}$ (Straizys \& Kuriliene, 1981). The mass of the invisible companion could thus be as large as $0.95 \pm 0.96 \mathrm{M}_{\odot}$. Unfortunately the corresponding uncertainty prevents us from drawing any conclusion about the nature of the invisible companion.

The mass of the $\mathrm{b}$ component can also be estimated with the measurement of angular separation of $\mathrm{Bb}$ made by McCarthy et al (1982), which was $\rho=0 .{ }^{\prime \prime} 17$ in 1981.94. The ephemerides for this epoch with our orbit Bb-P lead to $\rho^{\prime}=0^{\prime \prime} .056$. Since $\rho / \rho^{\prime}=\left(M_{B}+M_{b}\right) / M_{b}$, it comes: $M_{B} / M_{b}=2.0$ and $M_{b}=0.65 \mathrm{M}_{\odot}$. Hence the b component could be a late-type main sequence star of type K3-K5. McCarthy et al (1982) measured its flux in near infrared and found $K=6.0 \mathrm{mag}$ with a strong infrared excess $K-L=1.3 \pm 0.2$ mag, redder than that expected of even the coolest main-sequence stars and corresponding to a colour temperature of $1440 \mathrm{~K}$. It is thus expected to be much fainter than the A, B components, $\left(\Delta m_{V}>4\right.$ mag. $)$ which well explains the absence of detection with visual observations.

\section{Conclusion}

In the second semester of 2007, we obtained 283 new measurements of 279 visual binaries with PISCO in Merate, with an average accuracy of $0^{\prime \prime} .014$ for the angular separation and $0^{\circ} .6$ for the position angles. The total number of measurements made in Merate since 2004 now approaches 1600 . This work is thus a good contribution to the continuing monitoring of long period visual binary systems, which is important for refining systemic stellar masses.

We obtained new orbital elements of the perturbation orbit Bb-P of Zeta Aqr and were able, for the first time, to compute their errors. Infrared observations with a $4-m$ class telescope would be very useful to constrain this orbit, or more basically to provide a clear confirmation of the presence of the invisible companion at the right position.

Acknowledgements. We thank the members of the United States Naval Observatory, Washington DC, for kindly sending on request some lists of measurements of visual binaries. 
Table 2 New elements of the visual orbit AB and perturbation orbit Bb-P of ADS 15971.

\begin{tabular}{|c|c|c|c|c|c|c|c|c|c|c|c|c|}
\hline Orbit & $\begin{array}{c}\Omega_{2000} \\
\left({ }^{\circ}\right)\end{array}$ & $\begin{array}{l}\omega \\
\left({ }^{\circ}\right)\end{array}$ & $\begin{array}{c}i \\
\left(^{\circ}\right)\end{array}$ & $e$ & $\begin{array}{c}T \\
(\mathrm{yr})\end{array}$ & $\begin{array}{c}P \\
(\mathrm{yr})\end{array}$ & $\begin{array}{c}n \\
\left({ }^{\circ} / \mathrm{yr}\right)\end{array}$ & $\begin{array}{c}a \\
\left({ }^{\prime \prime}\right)\end{array}$ & $\begin{array}{c}\mathrm{A} \\
\left({ }^{\prime \prime}\right)\end{array}$ & $\begin{array}{c}\mathrm{B} \\
\left({ }^{\prime \prime}\right)\end{array}$ & $\begin{array}{c}\mathrm{F} \\
\left({ }^{\prime \prime}\right)\end{array}$ & $\begin{array}{c}\mathrm{G} \\
\left({ }^{\prime \prime}\right)\end{array}$ \\
\hline $\mathrm{AB}$ & 133.2 & 273.0 & 141.7 & $\overline{0.343}$ & 1982.733 & 486.70 & 0.7397 & 3.380 & -2.05206 & -1.68435 & -2.20940 & 2.55557 \\
\hline & \pm 3.4 & \pm 9.4 & \pm 1.0 & \pm 0.029 & \pm 4.2 & \pm 40 & \pm 0.061 & \pm 0.023 & & & & \\
\hline $\mathrm{Bb}-\mathrm{P}$ & $\begin{array}{c}20.900 \\
+26\end{array}$ & $\begin{array}{c}330.3 \\
+29\end{array}$ & $\begin{array}{r}22.3 \\
+10\end{array}$ & $\begin{array}{c}0.125 \\
+0.018\end{array}$ & $\begin{array}{c}2003.404 \\
+0.652\end{array}$ & $\begin{array}{l}25.822 \\
+0.139\end{array}$ & $\begin{array}{c}13.942 \\
+0.0751\end{array}$ & $\begin{array}{c}0.062 \\
+0.012\end{array}$ & 0.06045 & -0.00734 & 0.01092 & 0.05751 \\
\hline
\end{tabular}
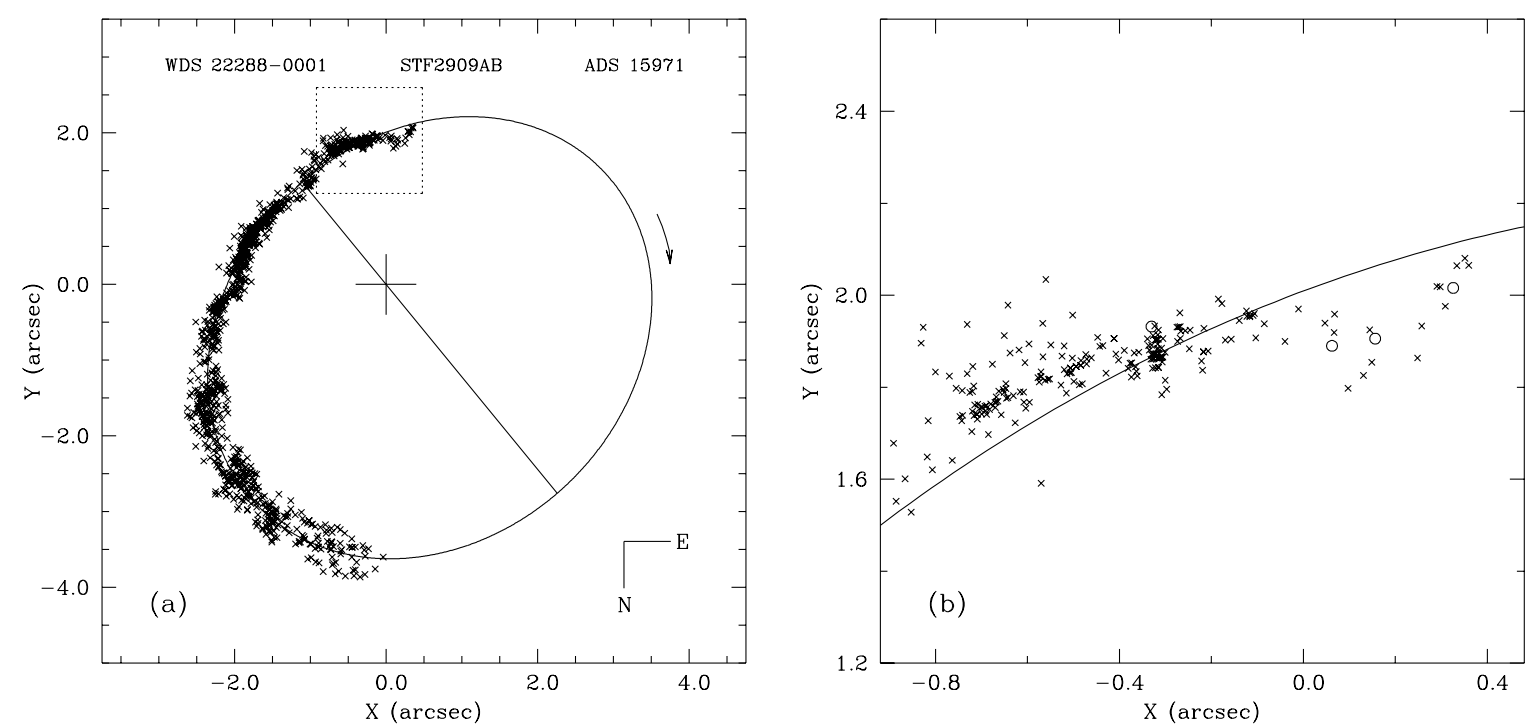

Fig. 5 New orbit of ADS 15971 AB (a) with enlarged part in (b), corresponding to the dotted square in (a). Plotted as a solid line, it is actually the orbit of the center of mass of the Bb couple relative to the component $\mathrm{A}$ marked as a big cross. The four observations of PISCO, including the one made at Pic du Midi by Scardia et al. (2000a) on 1998.879 (the first from left), are printed as circles.

Table 3 New ephemeris of the perturbation orbit Bb-P of ADS 15971. Here $\rho^{\prime}$ is the angular separation between $\mathrm{B}$ and the center of mass of the $\mathrm{Bb}$ system. The angular separation of $\mathrm{Bb}$ is estimated at $3.0 \rho^{\prime}$ (see text).

\begin{tabular}{cccccc}
\hline Epoch & $\begin{array}{c}\rho^{\prime} \\
\left({ }^{\prime \prime}\right)\end{array}$ & $\begin{array}{c}\theta \\
\left({ }^{\circ}\right)\end{array}$ & Epoch & $\begin{array}{c}\rho^{\prime} \\
\left(^{\prime \prime}\right)\end{array}$ & $\begin{array}{c}\theta \\
\left({ }^{\circ}\right)\end{array}$ \\
\hline 2009.0 & 0.058 & 81.7 & 2015.0 & 0.067 & 159.0 \\
2010.0 & 0.059 & 96.2 & 2016.0 & 0.067 & 169.8 \\
2011.0 & 0.060 & 110.1 & 2017.0 & 0.069 & 180.2 \\
2012.0 & 0.062 & 123.4 & 2018.0 & 0.069 & 190.5 \\
2013.0 & 0.064 & 135.9 & 2019.0 & 0.068 & 200.9 \\
2014.0 & 0.065 & 147.8 & 2020.0 & 0.067 & 211.6 \\
\hline
\end{tabular}

This work has made use of the "Fourth Catalogue of Interferometric Measurements of Binary Stars" (http://ad.usno. navy.mil/wds/int4), the "Sixth Catalogue of Orbits of Visual Binary Stars" (http://ad.usno.navy.mil/wds/orb6), the Washington Double Star Catalogue maintained at the U.S. Naval Observatory (http://ad.usno.navy.mil/wds/wds) and the SIMBAD data base (http://simbad.u-strasbg.fr/simba operated by the Centre de Données Astronomiques de Strasbourg (France).

\section{References}

Argyle, R. W.: 2009, Webb Society Double Star Section Circulars, 17

Aristidi, E., Carbillet, M., Lyon, J.-F., Aime, C.: 1997, A\&AS, 125, 139

Brendley, M. Hartkopf, W.I.: 2007, IAU Commission 26, Inf. Circ. 163

Couteau, P.: 1973, A\&AS 12, 127

Cvetkovic, Z.: 2007, IAU Commission 26, Inf. Circ. 162

Docobo, J.A., Costa, J.M.: 1984, IAU Commission 26, Inf. Circ. 92

Docobo, J.A., Costa, J.M.: 1990a, IAU Commission 26, Inf. Circ. 110

Docobo, J.A., Costa, J.M.: 1990b, PASP 102, 1400

Docobo, J.A., Ling, J.F.: 2007, AJ 133, 1209

Docobo, J.A., Ling, J.F.: 2008, IAU Commission 26, Inf. Circ. 164

Douglass, G.G., Hindsley, R.B., Worley, C.E.: 1997, ApJS $111,289,1997$

Franz, O.G.: 1958, AJ, 63, 329

Harrington, R.S.: 1968, AJ, 73, 508

Hartkopf, W.I., McAlister, H.A., Franz, O.G.: 1989, AJ 98, ad) 1014

Hartkopf, W.I., Mason, B.D.: 2001, IAU Commission 26, Inf. Circ. 145 

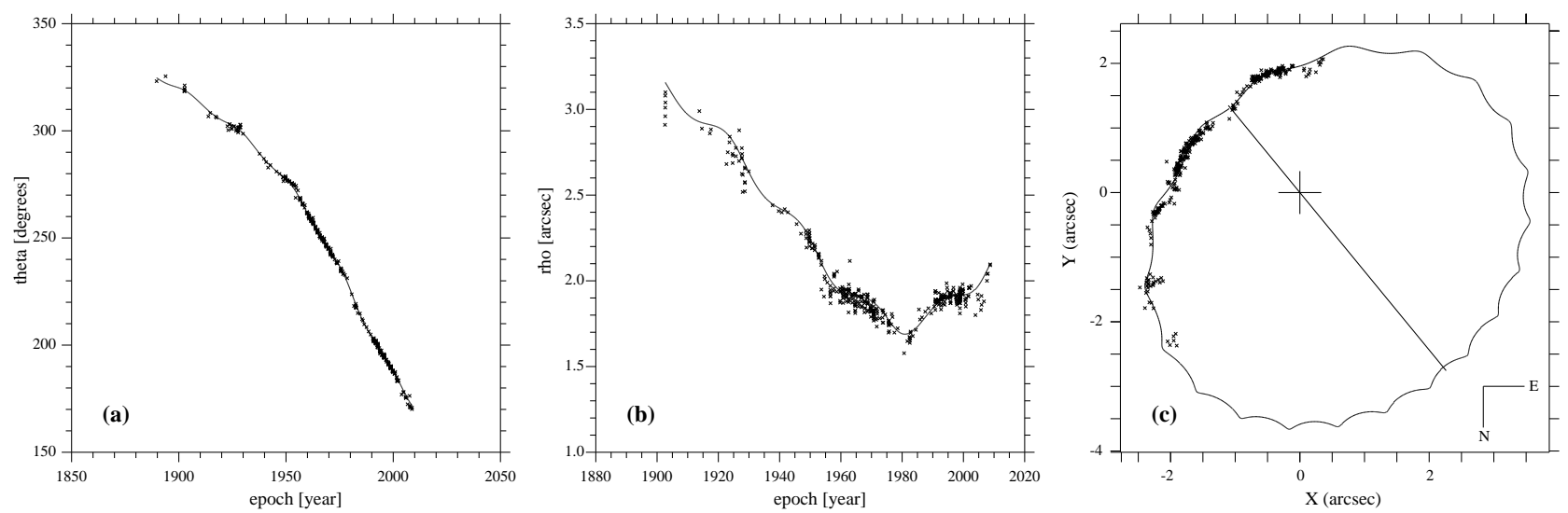

Fig. 6 New orbit of ADS 15971: combination of the AB and Bb-P orbits (solid line) compared to all the photographic and speckle measurements (crosses): (a) position angle vs epoch, (b) angular separation vs epoch, and (c) orbit in the plane of the sky.

Table 4 ADS 15971: O-C residuals obtained with the combination of the orbits $\mathrm{AB}$ and $\mathrm{Bb}-\mathrm{P}$. The symbol ${ }^{P}$ indicates PISCO measurements. Measurements with "Ary" in Col. 4 were made by RWA with a $20 \mathrm{~cm}$ refractor.

\begin{tabular}{crrl}
\hline Epoch & $\Delta \rho(\mathrm{O}-\mathrm{C})$ & $\Delta \theta(\mathrm{O}-\mathrm{C})$ & Observer \\
& $\left({ }^{\prime \prime}\right)$ & 0.511 & WSI \\
\hline 2002.531 & 0.039 & -0.031 & Ary \\
2002.800 & 0.006 & 0.621 & Alz \\
2003.740 & -0.049 & -0.147 & Ary \\
2003.804 & 0.020 & -3.273 & Dal \\
2003.937 & -0.152 & -0.680 & WSI \\
2004.669 & -0.048 & 0.082 & Alz \\
2004.750 & -0.030 & -0.418 & Ary \\
2004.800 & -0.012 & $-0.161^{P}$ & Paper II \\
2004.878 & $-0.083^{P}$ & -0.700 & WSI \\
2005.732 & -0.167 & -0.815 & Ary \\
2005.777 & -0.069 & $-0.985^{P}$ & Paper IV \\
2005.899 & $-0.090^{P}$ & -0.814 & Dal \\
2005.937 & -0.143 & -2.431 & WSI \\
2006.700 & -0.148 & -2.273 & Alz \\
2006.790 & -0.081 & -3.038 & Ary \\
2006.810 & 0.008 & -050 & Cli \\
2007.639 & -0.064 & -2.052 & WSI \\
2007.700 & -0.027 & -1.220 & Smr \\
2007.783 & -0.027 & Ary \\
2007.835 & 0.041 & -2.537 & This paper \\
2007.966 & $-0.031^{P}$ & $-1.932^{P}$ & Ary \\
2008.701 & 0.019 & -2.028 & Ant \\
2008.734 & -0.006 & -1.481 & Ant \\
2008.734 & -0.011 & -0.781 & Ant \\
\hline & & &
\end{tabular}

Hartkopf, W.I., Mason, B.D., Rafferty, T.: 2008, AJ 135, 1334

Hartkopf, W.I., Mason, B.D.: 2009, "Sixth Catalogue of Orbits of Visual Binary Stars" http://ad.usno.navy.mil/wds/orb6.html (OC6)

Hartkopf, W.I., Mason, B.D., Wycoff, G.L., McAlister, H.A.: 2009, "Fourth Catalogue of Interferometric Measurements of Binary Stars" http://ad.usno.navy.mil/wds/int4.html (IC4)
Table 5 New ephemeris of ADS 15971 - STF 2909 AB obtained with the combination of the $\mathrm{AB}$ and $\mathrm{Bb}-\mathrm{P}$ orbits.

\begin{tabular}{cccccc}
\hline Epoch & $\begin{array}{c}\rho \\
\left({ }^{\prime \prime}\right)\end{array}$ & $\begin{array}{c}\theta \\
\left({ }^{\circ}\right)\end{array}$ & Epoch & $\begin{array}{c}\rho \\
\left({ }^{\prime \prime}\right)\end{array}$ & $\begin{array}{c}\theta \\
\left({ }^{\circ}\right)\end{array}$ \\
\hline 2009.0 & 2.113 & 171.2 & 2015.0 & 2.313 & 164.7 \\
2010.0 & 2.151 & 169.9 & 2016.0 & 2.337 & 163.9 \\
2011.0 & 2.188 & 168.7 & 2017.0 & 2.358 & 163.0 \\
2012.0 & 2.223 & 167.6 & 2018.0 & 2.375 & 162.1 \\
2013.0 & 2.256 & 166.6 & 2019.0 & 2.389 & 161.2 \\
2014.0 & 2.286 & 165.6 & 2020.0 & 2.401 & 160.3 \\
\hline
\end{tabular}

Table 6 Physical parameters of Zeta Aqr AB ( $a$ and $\left.\mathfrak{M}_{\text {total }}\right)$ derived from the new orbital elements.

\begin{tabular}{llrrrr}
\hline $\mathrm{m}_{V}$ & Spec. type & $\begin{array}{r}\pi_{H I P} \\
(\mathrm{mas})\end{array}$ & $\left(^{\prime \prime}\right)$ & $\begin{array}{l}a \\
(\mathrm{AU})\end{array}$ & $\begin{array}{r}\mathfrak{M}_{\text {total }} \\
\mathrm{M}_{\odot}\end{array}$ \\
\hline 3.65 & F3V+F6IV & 35.68 & 3.38 & 94.7 & 3.6 \\
& & \pm 1.24 & \pm 0.02 & \pm 3.4 & \pm 1.0 \\
\hline
\end{tabular}

Heintz, W.D.: 1963, Veroff. Sternw. Munchen 5, 247-268

Heintz, W.D.: 1965, Veroff. Sternw. Munchen 7, 7-14

Heintz, W.D.: 1975, ApJS 29, 331

Heintz, W.D.: 1976, ApJ 208, 474

Heintz, W.D.: 1981, PASP 93, 90

Heintz, W.D.: 1984a, ApJ, 284, 806

Heintz, W.D.: 1984b, A\&AS 56, 5

Heintz, W.D.: 1984c, ApJ, 284, 806

Heintz, W.D.: 1991, A\&AS 90, 311

Heintz, W.D., Strom, C.: 1993, PASP 105, 293

Heintz, W.D.: 1996, ApJS 105, 475

Heintz, W.D.: 1997, ApJS 111, 335

Heintz, W.D.: 2001, IAU Commission 26, Inf. Circ. 143

Hellerich, J.: 1925, Astron. Nachr. 223, 335

Høg, E., Fabricius, C., Makarov, V.V., Urban, S., Corbin, T., Wycoff, G., Bastian, U., Schwekendiek, P., Wicenec, A.: 2000, Tycho-2 Cat., A\&A 357, 367

Hopmann, J.: 1964, Ann. Sternw. Wien 26, \#1

Kowalsky, M.: 1873, Procès-verbaux de l'Université Imperiale de Kasan

Ling, J.F.: 2008, IAU Commission 26, Inf. Circ. 164 
Mason, B.D., Hartkopf, W.I., Wycoff, G.L., Pascu, D., Urban, S.E., Hall, D.M., Hennessy, G.S., Rafferty, T.J., et al.: 2004a, AJ 127, 539

Mason, B.D., Hartkopf, W.I., Wycoff, G.L., Rafferty, T.J., Urban, S.E., Flagg, L.: 2004b, AJ 128, 3012

Mason, B.D., Hartkopf, W.I., Wycoff, G.L., Holdenried, E.R., 2006: AJ 132, 2219

Mason, B.D., Wycoff, G.L., Hartkopf, W.I.: 2009, "Washington Double Star Catalogue" http://ad.usno.navy.mil/wds/wds.html (WDS)

McCarthy, D.W., Low, F.J., Kleinmann, S.G., Arganbright, D.V.: 1982, ApJ, 257, L75-78

Novakovic, B., Todorovic, N.: 2006, Serbian AJ 172, 21

Novakovic, B.: 2007, Chin. J. Astron. Astrophys. 7, 415

Novakovic, B.: 2008a, IAU Commission 26, Inf. Circ. 165

Novakovic, B.: 2008b, Observatory 128, 56

Olevic, D., Popovic, G.M., Zulevic, D.J., Catovic, Z.: 1993, Bull. Obs. Astron. Belgrade \#148, 49

Olevic, D., Jovanovic, P.: 2001, Serbian AJ 163, 5

Olevic, D., Cvetkovic, Z.: 2004, IAU Commission 26, Inf. Circ. 152

Popovic, G.M.: 1969, Bull. Obs. Astron. Belgrade 27, \#1, 33-54

Popovic, G.M., Pavlovic, R., Zivkov, V.: 2000, A\&AS 144, 211

Pourbaix, D.: 2000, A\&AS 145, 215

Prieur, J.-L, Koechlin, L., André, C., Gallou, G., Lucuix, C.: 1998, Experimental Astronomy, vol 8, Issue 4, 297

Prieur, J.-L., Scardia, M., Pansecchi, L., Argyle, R.W., Sala, M., Ghigo, M., Koechlin, L., Aristidi, E.: 2008, MNRAS, 387, 772 (Paper V)

Prieur, J.-L., Scardia, M., Pansecchi, L., Argyle, R.W., Sala, M.: 2009, MNRAS, 395, 907 (Paper VII)

Scardia, M.: 1983, IAU Commission 26, Inf. Circ. 89

Scardia, M., Prieur, J.-L., Aristidi, E., Koechlin, L.: 2000a, ApJS, 131, 561

Scardia, M., Prieur, J.-L., Aristidi, E., Koechlin, L.: 2000b, AN 321, 255

Scardia, M., Prieur, J.-L., Koechlin, L., Aristidi, E.: 2001, AN 322, 161

Scardia, M., Prieur, J.-L., Koechlin, L., Aristidi, E.: 2002, IAU Commission 26, Inf. Circ. 148

Scardia, M., Prieur, J.-L., Sala, M., Ghigo, M., Koechlin, L., Aristidi, E., Mazzoleni, F.: 2005, MNRAS, 357, 1255 (with erratum in MNRAS 362, 1120) (Paper I)

Scardia, M., Prieur, J.-L., Pansecchi, L., Argyle, R.W., Sala, M., Ghigo, M., Koechlin, L., Aristidi, E.: 2006, MNRAS, 367, 1170 (Paper II)

Scardia, M., Prieur, J.-L., Pansecchi, L., Argyle, R.W., Basso, S., Sala, M., Ghigo, M., Koechlin, L., Aristidi, E.: 2007, MNRAS, 374, 965 (Paper III)

Scardia, M., Prieur, J.-L., Pansecchi, L., Argyle, R.W., Sala, M., Basso, S., Ghigo, M., Koechlin, L., Aristidi, E.: 2008a, AN 329, 54

Scardia, M., Prieur, J.-L., Pansecchi, L., Argyle, R.W., Sala, M., Basso, S., Ghigo, M., Koechlin, L., Aristidi, E.: 2008b, Astron. Nach., 329, 1, 54 (Paper IV)

Scardia, M., Prieur, J.-L., Pansecchi, L., Argyle, R.W.: 2008c, AN 329, 379

Scardia M., Prieur J.-L., Pansecchi L., Argyle R.W., Sala M.: 2009, Astron. Nach., 330, 1, 55 (Paper VI)

Seymour, D., Mason, B.D., Hartkopf, W.I., Wycoff, G.L.: 2002, AJ 123, 1023
Söderhjelm, S.: 1999, A\&A 341, 121

Starikova, G.A.: 1977, Soobchen. Gos. Astr. Inst. Sternberg \#199, 12

Starikova, G.A.: 1982, SvAL 8, 166 (1982, Pisma Astron. Zhur. 8, 306)

Strand, K.A.: 1942, AJ, 49, 165

Straizys, V., Kuriliene, G.: 1981, Astrophys. Space Sci. 80, 353

Tokovinin, A.A.: 1984, SvAL 10, 121

Valbousquet, A.: 1981, IAU Commission 26, Inf. Circ. 83

van Leeuwen, F.: 2007, "Hipparcos, the new reduction of the raw data" (Dordrecht: Springer)

Weigelt, G.: 1983, Lowell Observatory Bulletin 167, 144

Zulevic, D.J.: 1977, IAU Commission 26, Inf. Circ. 72 